\title{
COMPOUND-COMMUTING ADDITIVE MAPS ON MATRIX SPACES
}

\author{
WAi LeONG ChOOI
}

\begin{abstract}
In this note, compound-commuting additive maps on matrix spaces are studied. We show that compound-commuting additive maps send rank one matrices to matrices of rank less than or equal to one. By using the structural results of rank-one nonincreasing additive maps, we characterize compound-commuting additive maps on four types of matrices: triangular matrices, square matrices, symmetric matrices and Hermitian matrices.
\end{abstract}

\section{Introduction}

Let $\mathcal{M}_{1}$ and $\mathcal{M}_{2}$ be matrix spaces over the same field. Let $\Phi$ be a matrix function such that $\Phi(A) \in \mathcal{M}_{i}$ whenever $A \in \mathcal{M}_{i}$ for $i=1,2$. We say that a map $\psi: \mathcal{M}_{1} \rightarrow \mathcal{M}_{2}$ is $\Phi$-commuting if $(\psi \circ \Phi)(A)=(\Phi \circ \psi)(A)$ for all $A \in \mathcal{M}_{1}$. In 1982, Sinkhorn [13] first studied $\Phi$-commuting linear maps on $n$-square complex matrices with $\Phi(A)=\operatorname{adj} A$, where $\operatorname{adj} A$, the adjugate of $A$, is the matrix whose $(i, j)$-th entry is the cofactor of the row $j$ and column $i$ of $A$. By using the classical theorem of Frobenius [6] concerning determinant linear preservers, he gave a general form of adjugate-commuting linear maps on $n$-square complex matrices and showed that if $n \geqslant 3$, then the map is of the form

$$
A \mapsto \lambda P A P^{-1} \quad \text { or } \quad A \mapsto \lambda P A^{t} P^{-1},
$$

where $\lambda \in \mathbb{C}$ with $\lambda^{n-2}=1, P$ is an invertible $n$-square complex matrix, and $A^{t}$ denotes the transpose of $A$. This result was generalized by Chan et al. in [2] to arbitrary infinite fields based on the structural results of linear maps preserving invertibility. In the same paper, linear maps on the spaces of square matrices and symmetric matrices that commute with the exponential function, i.e., $\Phi(A)=e^{A}$, were also considered. Later on, Chan and Lim [1] characterized linear maps on square matrices that commute with the $k$-th power function,

Received April 10, 2009; Revised May 19, 2010.

2010 Mathematics Subject Classification. 15A03, 15A04.

Key words and phrases. rank, compound matrix, compound-commuting additive map, rank-one nonincreasing additive map.

The author's research was partially supported by FRGS National Research Grant FS273/2008A and Short-term Grant FS276/2008C. 
i.e., $\Phi(A)=A^{k}$ for some fixed positive integer $k$. More recently, by relaxing the linearity assumption, some researchers considered $\Phi$-commuting additive maps. For instance, adjugate-commuting additive maps were studied on the space of complex Hermitian matrices in [14], square matrices and symmetric matrices in [15], and triangular matrices in [15, 3], respectively. We refer the reader to [12, Chapter 9] and [17, Chapter 10] for more information.

Let $\mathbb{F}$ be a field and let $\mathcal{M}_{m, n}(\mathbb{F})$ denote the linear space of $m \times n$ rectangular matrices over $\mathbb{F}$. We write $\mathcal{M}_{n, n}(\mathbb{F})$ as $\mathcal{M}_{n}(\mathbb{F})$. If $A \in \mathcal{M}_{n}(\mathbb{F})$, then the $(n-1)$ th compound matrix, or simply compound matrix of $A$, denoted by $C_{n-1}(A)$, is the $n$-square matrix whose entries are

$$
C_{n-1}(A)_{i j}=\operatorname{det}(A[n+1-i \mid n+1-j])
$$

with $1 \leqslant i, j \leqslant n$, where $A[i \mid j]$ is the $(n-1)$-square submatrix of $A$ obtained by excluding its $i$-th row and $j$-th column. In this note, we study $\Phi$-commuting additive maps in the context of $\Phi(A)=C_{n-1}(A)$, and call such a map compoundcommuting. Nevertheless, to our knowledge, compound-commuting additive maps on matrix spaces have not been studied yet. We show, in Lemma 2.5, that every nonzero compound-commuting additive map is rank-one nonincreasing, i.e., the map sends rank one matrices to matrices of rank less than or equal to one. By using the structural results of rank-one nonincreasing additive maps on the space of block triangular matrices in [3], symmetric matrices in [8,7], and Hermitian matrices in $[9,10,11]$, respectively, we characterize compoundcommuting additive maps on the spaces of square matrices, triangular matrices and symmetric matrices over arbitrary fields, and on the space of Hermitian matrices over a field with proper involution. We will see in Theorem 2.8 that the classification of compound-commuting additive maps on triangular matrices is quite different and essentially more complicated than the corresponding theorems on spaces of square matrices, symmetric matrices, and Hermitian matrices.

We introduce some notation that will be needed in our discussion. Throughout this note, unless otherwise specified, we assume $\mathbb{F}$ is an arbitrary field, and $m$ and $n$ are positive integers with $n \leqslant m$. Let $k, n_{1}, \ldots, n_{k}$ be positive integers satisfying $n_{1}+\cdots+n_{k}=n$. By $\mathcal{T}_{n_{1}, \ldots, n_{k}}(\mathbb{F})$, we designate the subalgebra of $\mathcal{M}_{n}(\mathbb{F})$ consisting of block matrices $\left(A_{i j}\right)$ of the form

$$
\left(\begin{array}{cccc}
A_{11} & A_{12} & \cdots & A_{1 k} \\
0 & A_{22} & \cdots & A_{2 k} \\
\vdots & \vdots & \ddots & \vdots \\
0 & 0 & \cdots & A_{k k}
\end{array}\right)
$$

with $A_{i j} \in \mathcal{M}_{n_{i}, n_{j}}(\mathbb{F})$ for every $1 \leqslant i \leqslant j \leqslant k$. We shall call such an algebra $\mathcal{T}_{n_{1}, \ldots, n_{k}}(\mathbb{F})$ a block triangular matrix algebra. In particular, when $n_{i}=1$ for all $i$, then it constitutes the algebra of $n$-square triangular matrices and is abbreviated to $\mathcal{T}_{n}(\mathbb{F})$, and $\mathcal{T}_{n_{1}, \ldots, n_{k}}(\mathbb{F})=\mathcal{M}_{n}(\mathbb{F})$ when $k=1$. For each block triangular matrix algebra $\mathcal{T}_{n_{1}, \ldots, n_{k}}(\mathbb{F})$, we associate a chain of nonnegative 
integers $0=\delta_{0}<\delta_{1}<\cdots<\delta_{k-1}<\delta_{k}=n$ with $\delta_{i}=n_{1}+\cdots+n_{i}$ for each $i=1, \ldots, k$. Let ${ }^{-}: \mathbb{F} \rightarrow \mathbb{F}$ be a field involution (i.e., $\overline{a+b}=\bar{a}+\bar{b}, \overline{a b}=\bar{b} \bar{a}$, and $\overline{\bar{a}}=a$ for every $a, b \in \mathbb{F})$. A matrix $A \in \mathcal{M}_{n}(\mathbb{F})$ is symmetric if $A^{t}=A$, and is called a Hermitian matrix on the involution - of $\mathbb{F}$, or simply Hermitian, if $A^{*}:=\bar{A}^{t}=A$, where ${ }^{-}$is applied on $A$ entrywise. Let $\mathcal{S}_{n}(\mathbb{F})$ and $\mathcal{H}_{n}(\mathbb{F})$ denote, respectively, the set of all $n$-square symmetric matrices and $n$-square Hermitian matrices over $\mathbb{F}$. Note that $\mathcal{H}_{n}(\mathbb{F})=\mathcal{S}_{n}(\mathbb{F})$ if the involution - is identity. We will use $E_{i j}$ and $F_{i j}$ to designate the matrix units of $\mathcal{M}_{n}(\mathbb{F})$ and $\mathcal{M}_{m}(\mathbb{F})$, respectively, whose $(i, j)$-th entry is one and the others are zero. We denote by $I_{n}$ the $n$-square identity matrix, and $Z_{n}$ the diagonal matrix $\sum_{i=1}^{n}(-1)^{i+1} E_{i i}$. Given a matrix $A=\left(a_{i j}\right) \in \mathcal{M}_{m, n}(\mathbb{F})$, by $\operatorname{rank} A, A^{\sigma}$ and $A^{\sim}$, we denote, respectively, the $\operatorname{rank}$ of $A$, the $m \times n$ matrix whose $(i, j)$-th entry is $\sigma\left(a_{i j}\right)$ with $\sigma$ a field homomorphism on $\mathbb{F}$, and the $n \times m$ matrix whose $(i, j)$-th entry is $a_{m+1-j, n+1-i}$. It is easily verified that $A^{\sim}=J_{n} A^{t} J_{m}$ where $J_{n}:=E_{n 1}+E_{n-1,2}+\cdots+E_{1 n}$.

\section{Results}

In this section we characterize compound-commuting additive maps on the space of square matrices, triangular matrices, symmetric matrices, and Hermitian matrices, respectively. For the construction of our main results, we establish the following lemmas.

We start with the following interesting result proved by Fošner and Šemrl in $[5]$.

Lemma 2.1 ([5, Lemma 2.1]). Let $\mathbb{F}$ be a field, and let $m$ and $n$ be positive integers with $m>n$. If $A_{1}, \ldots, A_{m}$ are matrices in $\mathcal{M}_{n}(\mathbb{F})$ such that $\operatorname{det}\left(\sum_{i \in I} A_{i}\right)=0$ for every nonempty proper subset $I$ of $\{1, \ldots, m\}$, then $\operatorname{det}\left(A_{1}+\cdots+A_{m}\right)=0$.

We recall from the introduction that

$$
J_{n}=\sum_{i=1}^{n} E_{n+1-i, i} \quad \text { and } \quad Z_{n}=\sum_{i=1}^{n}(-1)^{i+1} E_{i i} .
$$

The proofs of the following Lemmas 2.2 and 2.3 are standard and will be omitted.

Lemma 2.2. Let $\mathbb{F}$ be a field, and let $n$ be an integer with $n \geqslant 2$. Then the following hold true.

(i) $C_{n-1}\left(I_{n}\right)=I_{n}$ and $C_{n-1}(0)=0$.

(ii) If $A=J_{n}$ or $A=Z_{n}$, then

$$
C_{n-1}(A)=\left\{\begin{array}{cc}
-A & \text { if } n=4 k-1,4 k, \text { for } k=1,2, \ldots, \\
A & \text { otherwise. }
\end{array}\right.
$$

(iii) Let $a \in \mathbb{F}$. Then 
(a) $C_{n-1}\left(I_{n}-E_{n+1-i, n+1-i}+(a-1) E_{j j}\right)=a E_{i i}$ for every $1 \leqslant i \leqslant n$, where $j$ is an integer with $1 \leqslant j \neq n+1-i \leqslant n$.

(b) $C_{n-1}\left(I_{n}-E_{n+1-i, n+1-i}-E_{n+1-j, n+1-j}+(-1)^{i+j+1} a E_{n+1-j, n+1-i}\right)$ $=a E_{i j}$ for every $1 \leqslant i \neq j \leqslant n$.

Lemma 2.3. Let $\mathbb{F}$ be a field, and let $n$ be an integer with $n \geqslant 2$. Let $\sigma$ : $\mathbb{F} \rightarrow \mathbb{F}$ be a field homomorphism and let $-: \mathbb{F} \rightarrow \mathbb{F}$ be a field involution. Let $A, B \in \mathcal{M}_{n}(\mathbb{F})$ and $\alpha \in \mathbb{F}$. Then the following statements hold true.

(i) $C_{n-1}(\alpha A)=\alpha^{n-1} C_{n-1}(A)$.

(ii) $C_{n-1}(A B)=C_{n-1}(A) C_{n-1}(B)$.

(iii) $C_{n-1}\left(A^{-1}\right)=C_{n-1}(A)^{-1}$ when $A$ is invertible.

(iv) $C_{n-1}\left(A^{\sigma}\right)=C_{n-1}(A)^{\sigma}$.

(v) $C_{n-1}\left(A^{t}\right)=C_{n-1}(A)^{t}$.

(vi) $C_{n-1}\left(A^{\sim}\right)=C_{n-1}(A) \sim$.

(vii) $C_{n-1}(\bar{A})=\overline{C_{n-1}(A)}$.

(viii) $\operatorname{rank} C_{n-1}(A)=n$ whenever $\operatorname{rank} A=n$.

(ix) $\operatorname{rank} C_{n-1}(A)=1$ whenever $\operatorname{rank} A=n-1$.

(x) $\operatorname{rank} C_{n-1}(A)=0$ whenever $\operatorname{rank} A \leqslant n-2$.

(xi) $C_{n-1}(A)=Z_{n}\left((\operatorname{adj} A)^{\sim}\right) Z_{n}$.

Lemma 2.4. Let $\mathbb{F}$ be a field and $\mathbb{K}$ be a field with an involution - . Let $n$ be an integer with $n \geqslant 2$. Let $\mathcal{S}=\mathcal{T}_{n_{1}, \ldots, n_{k}}(\mathbb{F})$ or $\mathcal{H}_{n}(\mathbb{K})$. If $A \in \mathcal{S}$ is of rank one, then there is a rank $n-1$ matrix $B \in \mathcal{S}$ such that $A=C_{n-1}(B)$.

Proof. Let $A \in \mathcal{S}$ be a rank one matrix. We first claim that there exists a rank $n-1$ matrix $D \in \mathcal{S}$ such that $A=\operatorname{adj} D$. We distinguish our proof into the following two cases.

Case I: $\mathcal{S}=\mathcal{T}_{n_{1}, \ldots, n_{k}}(\mathbb{F})$. Then there exist $E_{s t} \in \mathcal{T}_{n_{1}, \ldots, n_{k}}(\mathbb{F})$ and invertible matrices $P_{1}, P_{2} \in \mathcal{T}_{n_{1}, \ldots, n_{k}}(\mathbb{F})$ such that $A=P_{1} E_{s t} P_{2}$ (see [4, Lemma 2.1]). Let $\alpha_{i}=\left(\operatorname{det} P_{i}\right)^{n-2}$ and let $Q_{i}=\operatorname{adj} P_{i}$ for $i=1,2$. Clearly, $\alpha_{1}, \alpha_{2} \in \mathbb{F}$ are nonzero and $Q_{1}, Q_{2} \in \mathcal{T}_{n_{1}, \ldots, n_{k}}(\mathbb{F})$ are invertible. We let

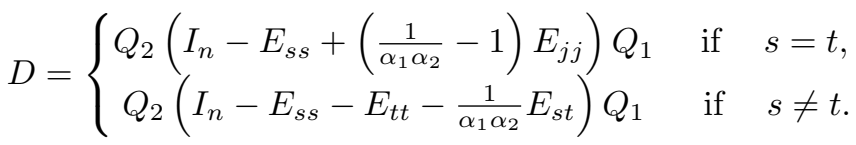

Here, $j$ is an integer with $1 \leqslant j \neq s \leqslant n$. It is clear that $D \in \mathcal{T}_{n_{1}, \ldots, n_{k}}(\mathbb{F})$ is of rank $n-1$. Since $\operatorname{adj} Q_{i}=\left(\operatorname{det} P_{i}\right)^{n-2} P_{i}=\alpha_{i} P_{i}$ for $i=1,2$, it follows that $\operatorname{adj} D=\left(\alpha_{1} P_{1}\right)\left(\frac{1}{\alpha_{1} \alpha_{2}} E_{s t}\right)\left(\alpha_{2} P_{2}\right)=P_{1} E_{s t} P_{2}=A$.

Case II: $\mathcal{S}=\mathcal{H}_{n}(\mathbb{K})$. If $A \in \mathcal{S}$ is of rank one, then there exist an invertible matrix $Q \in \mathcal{M}_{n}(\mathbb{K})$ and a scalar $\lambda \in \mathbb{K}$ with $\bar{\lambda}=\lambda$ such that $A=\lambda Q E_{11} Q^{*}$ $\left(\right.$ see $\left[16\right.$, Proposition 1.32]). Let $\beta=(\operatorname{det} Q)^{n-2}(\overline{\operatorname{det} Q})^{n-2}$. It is clear that $\bar{\beta}=\beta \neq 0$. Let $U=\operatorname{adj} Q$, and let

$$
D=U^{*}\left(I_{n}-E_{11}-E_{22}+\frac{\lambda}{\beta} E_{22}\right) U .
$$


It is clear that $D \in \mathcal{H}_{n}(\mathbb{K})$ because $\overline{\lambda / \beta}=\lambda / \beta$. Since $\operatorname{adj}\left(U^{*}\right)=(\operatorname{adj} U)^{*}$, we have

$$
\begin{aligned}
\operatorname{adj} D & =(\operatorname{adj} U) \operatorname{adj}\left(I_{n}-E_{11}-E_{22}+\frac{\lambda}{\beta} E_{22}\right)(\operatorname{adj} U)^{*} \\
& =(\operatorname{det} Q)^{n-2} Q\left(\frac{\lambda}{\beta} E_{11}\right) Q(\overline{\operatorname{det} Q})^{n-2} Q^{*}=\left(\lambda E_{11}\right) Q^{*}=A .
\end{aligned}
$$

Our claim is proved.

Let $B=Z_{n} D^{\sim} Z_{n}$. Evidently, $B$ is a rank $n-1$ matrix in $\mathcal{S}$, and

$$
C_{n-1}(B)=C_{n-1}\left(Z_{n} D^{\sim} Z_{n}\right)=C_{n-1}\left(Z_{n}\right) C_{n-1}\left(D^{\sim}\right) C_{n-1}\left(Z_{n}\right) .
$$

By the fact that $\operatorname{adj}\left(D^{\sim}\right)=(\operatorname{adj} D)^{\sim}$ and Lemma $2.3(\mathrm{xi})$, we have $C_{n-1}\left(D^{\sim}\right)=$ $Z_{n}(\operatorname{adj} D) Z_{n}$. By $(1)$ and Lemma $2.2(\mathrm{ii}), C_{n-1}(B)=Z_{n} C_{n-1}\left(D^{\sim}\right) Z_{n}=$ $Z_{n}\left(Z_{n}(\operatorname{adj} D) Z_{n}\right) Z_{n}=\operatorname{adj} D=A$, as desired.

Lemma 2.5. Let $\mathbb{F}$ be a field and let $\mathbb{K}$ be a field with an involution - . Let $m$ and $n$ be integers with $m, n \geqslant 3$. Let $\psi$ be a compound-commuting additive map from $\mathcal{T}_{n_{1}, \ldots, n_{k}}(\mathbb{F})$ into $\mathcal{M}_{m}(\mathbb{F})$ or from $\mathcal{H}_{n}(\mathbb{K})$ into $\mathcal{H}_{m}(\mathbb{K})$. Then $\psi$ is rank-one nonincreasing. Moreover, if $\psi \neq 0$, then $m=n$ and $\psi\left(I_{n}\right)$ is of rank $n$

Proof. Let $\mathcal{S}=\mathcal{T}_{n_{1}, \ldots, n_{k}}(\mathbb{F})$ or $\mathcal{H}_{n}(\mathbb{K})$. Let $A \in \mathcal{S}$ be a rank one matrix. We see that $\operatorname{rank} \psi(A) \neq m$ because $n \geqslant 3$ and $C_{n-1}(\psi(A))=\psi\left(C_{n-1}(A)\right)=\psi(0)=0$. By Lemma 2.4, there exists a rank $n-1$ matrix $B \in \mathcal{S}$ such that $A=C_{n-1}(B)$, and so, $\psi(A)=\psi\left(C_{n-1}(B)\right)=C_{n-1}(\psi(B))$. Since $\operatorname{rank} \psi(A) \neq m$, it follows that $\operatorname{rank} \psi(B) \leqslant m-1$, and hence, $\operatorname{rank} \psi(A) \leqslant 1$. Consequently, $\psi$ is a rank-one nonincreasing additive map.

Let $\psi$ be a nonzero map. Since $C_{n-1}\left(\psi\left(I_{n}\right)\right)=\psi\left(C_{n-1}\left(I_{n}\right)\right)=\psi\left(I_{n}\right)$, it follows from Lemma 2.3(viii)-(x) that either $\psi\left(I_{n}\right)=0$ or $\psi\left(I_{n}\right)$ is of rank $m$. Suppose $\psi\left(I_{n}\right)=0$. Then $\psi\left(E_{i i}\right)=0$ for all $i=1, \ldots, n$. This is because, for each $1 \leqslant i \leqslant n, \psi\left(E_{i i}\right)=\psi\left(C_{n-1}\left(I_{n}-E_{n+1-i, n+1-i}\right)\right)=C_{n-1}\left(\psi\left(I_{n}-\right.\right.$ $\left.\left.E_{n+1-i, n+1-i}\right)\right)=C_{n-1}\left(-\psi\left(E_{n+1-i, n+1-i}\right)\right)=0$. For each $a \in \mathbb{F}$ (respectively, $a \in \mathbb{K}$ with $\bar{a}=a$ if $\left.\mathcal{S}=\mathcal{H}_{n}(\mathbb{K})\right)$ and $E_{i i} \in \mathcal{S}$, in view of Lemma 2.2(iii), we see that $a E_{i i}=C_{n-1}\left(I_{n}-E_{n+1-i, n+1-i}+(a-1) E_{j j}\right)$, where $j$ is an integer with $1 \leqslant j \neq n+1-i \leqslant n$. Thus, $\psi\left(a E_{i i}\right)=C_{n-1}\left(\psi\left(I_{n}-E_{n+1-i, n+1-i}+(a-\right.\right.$ 1) $\left.\left.E_{j j}\right)\right)=C_{n-1}\left(\psi\left((a-1) E_{j j}\right)\right)=0$. Therefore, we have

$$
\psi\left(a E_{i i}\right)=0
$$

for every $E_{i i} \in \mathcal{S}$ and $a \in \mathbb{F}$ (respectively, $a \in \mathbb{K}$ with $\bar{a}=a$ if $\mathcal{S}=\mathcal{H}_{n}(\mathbb{K})$ ).

When $\mathcal{S}=\mathcal{T}_{n_{1}, \ldots, n_{k}}(\mathbb{F})$, for each $a \in \mathbb{F}$ and $E_{i j} \in \mathcal{T}_{n_{1}, \ldots, n_{k}}(\mathbb{F})$ with $1 \leqslant i \neq$ $j \leqslant n$, we have

$a E_{i j}=C_{n-1}\left(I_{n}-E_{n+1-i, n+1-i}-E_{n+1-j, n+1-j}+(-1)^{i+j+1} a E_{n+1-j, n+1-i}\right)$

by Lemma 2.2 (iii). So, by (2), we see that

$$
\psi\left(a E_{i j}\right)=C_{n-1}\left(\psi\left((-1)^{i+j+1} a E_{n+1-j, n+1-i}\right)\right)=0
$$


since $\psi$ is rank-one nonincreasing. Therefore, $\psi\left(a E_{i j}\right)=0$ for every $a \in \mathbb{F}$ and $E_{i j} \in \mathcal{T}_{n_{1}, \ldots, n_{k}}(\mathbb{F})$ with $1 \leqslant i \neq j \leqslant n$.

When $\mathcal{S}=\mathcal{H}_{n}(\mathbb{K})$, for each $a \in \mathbb{K}$ and $1 \leqslant i \neq j \leqslant n$, for the sake of simplicity, we denote

$$
\left.G=I_{n}-E_{n+1-i, n+1-i}-E_{n+1-j, n+1-j}+a E_{n+1-i, n+1-j}+\bar{a} E_{n+1-j, n+1-i}\right) .
$$

We see that $G \in \mathcal{H}_{n}(\mathbb{K})$ and $C_{n-1}(G)=-a \bar{a}\left(I_{n}-E_{i i}-E_{j j}\right)+(-1)^{i+j+1} \bar{a} E_{i j}+$ $a E_{j i}$. By (2), we obtain $\psi\left(C_{n-1}(G)\right)=\psi\left(-a \bar{a}\left(I_{n}-E_{i i}-E_{j j}\right)+(-1)^{i+j+1} \bar{a} E_{i j}+\right.$ $\left.a E_{j i}\right)=(-1)^{i+j+1} \psi\left(\bar{a} E_{i j}+a E_{j i}\right)$. On the other hand, by $(2)$, we see that

$$
\begin{aligned}
\psi\left(C_{n-1}(G)\right) & =C_{n-1}(\psi(G))=C_{n-1}\left(\psi\left(a E_{n+1-i, n+1-j}+\bar{a} E_{n+1-j, n+1-i}\right)\right) \\
& =\psi\left(C_{n-1}\left(a E_{n+1-i, n+1-j}+\bar{a} E_{n+1-j, n+1-i}\right)\right)=0 .
\end{aligned}
$$

Hence, $\psi\left(\bar{a} E_{i j}+a E_{j i}\right)=0$ for all $a \in \mathbb{K}$ and $1 \leqslant i \neq j \leqslant n$.

In both cases, together with (2), we conclude that $\psi=0$, which contradicts to our hypothesis. Then $\psi\left(I_{n}\right) \neq 0$. Hence, $\psi$ is a rank-one nonincreasing additive mapping with $\psi\left(I_{n}\right)$ being of rank $m$. By the additivity of $\psi$, we see that

$$
m=\operatorname{rank} \psi\left(I_{n}\right) \leqslant \sum_{i=1}^{n} \operatorname{rank} \psi\left(E_{i i}\right) \leqslant n .
$$

Suppose $n>m$. Since $\psi\left(I_{n}\right)$ is of rank $m$, it follows from Lemma 2.1 that there exist integers $1 \leqslant s_{1}<\cdots<s_{k} \leqslant n$ with $m \leqslant k \leqslant n-1$ such that $\operatorname{rank} \psi\left(E_{s_{1} s_{1}}+\cdots+E_{s_{k} s_{k}}\right)=m$. Thus,

$m=\operatorname{rank} C_{n-1}\left(\psi\left(E_{s_{1} s_{1}}+\cdots+E_{s_{k} s_{k}}\right)\right)=\operatorname{rank} \psi\left(C_{n-1}\left(E_{s_{1} s_{1}}+\cdots+E_{s_{k} s_{k}}\right)\right) \leqslant 1$, a contradiction. Hence, $m=n$. This completes our proof.

Let $n \geqslant 1$ and let $\mathbb{F}$ be a field. Let $r, \epsilon_{1}, \ldots, \epsilon_{r}$ be positive integers such that $\epsilon_{1}+\cdots+\epsilon_{r}=n$. For each integer $1 \leqslant i \leqslant r$, we denote $d_{i}=\epsilon_{1}+\cdots+\epsilon_{i}$ and $d_{0}=0$. Given a matrix $A \in \mathcal{M}_{n}(\mathbb{F})$, we associate a unique $n$-square block diagonal matrix

$$
\bigoplus_{i=1}^{r} A_{i}:=\left(\begin{array}{cccc}
A_{1} & 0 & \cdots & 0 \\
0 & A_{2} & \cdots & 0 \\
\vdots & \vdots & \ddots & \vdots \\
0 & 0 & \cdots & A_{r}
\end{array}\right)
$$

where, for each $1 \leqslant i \leqslant r, A_{i}$ is the $\epsilon_{i}$-square principal submatrix of $A$ lying in rows $\left(d_{i-1}+1\right), \ldots, d_{i}$ and columns $\left(d_{i-1}+1\right), \ldots, d_{i}$. Such a block matrix $\bigoplus_{i=1}^{r} A_{i}$ is called the $\left(\epsilon_{1}, \ldots, \epsilon_{r}\right)$-block diagonal matrix induced by $A$.

Given an $n$-square block triangular matrix algebra $\mathcal{T}_{n_{1}, \ldots, n_{k}}(\mathbb{F})$, we denote

$$
\Omega=\left\{\delta_{i}: n_{i}=1,1 \leqslant i \leqslant k\right\} \cup\{1, n\} .
$$

Let $h$ be a positive integer with $h \leqslant|\Omega|$. By $H_{h, n_{1}, \ldots, n_{k}}$, we designate the totality of strictly increasing sequences of $h$ integers $\alpha=\left(\alpha_{1}, \ldots, \alpha_{h}\right)$ chosen from $\Omega$ satisfying $\alpha_{1}=1$ and $\alpha_{h}=n$. In particular, when $n_{i}=1$ for all $i$, we write $H_{h, n_{1}, \ldots, n_{k}}$ as $H_{h, n}$. It is clear that when $n=1$ (i.e., $\mathcal{T}_{n_{1}, \ldots, n_{k}}(\mathbb{F})=$ 
$\left.\mathcal{T}_{1}(\mathbb{F})\right), \Omega=\{1\}$ and $\alpha=1$. Given $\alpha=\left(\alpha_{1}, \alpha_{2}, \ldots, \alpha_{h}\right) \in H_{h, n}$, we denote $\alpha^{\sim}=\left(n+1-\alpha_{h}, \ldots, n+1-\alpha_{2}, n+1-\alpha_{1}\right) \in H_{h, n}$, and clearly, $\left(\alpha^{\sim}\right)^{\sim}=\alpha$. For example, if $\alpha=(1,2,6,9) \in H_{4,9}$, then $\alpha^{\sim}=(1,4,8,9) \in H_{4,9}$.

For each matrix $A=\left(a_{i j}\right) \in \mathcal{T}_{n_{1}, \ldots, n_{k}}(\mathbb{F})$ and $\alpha=\left(\alpha_{1}, \ldots, \alpha_{h}\right) \in H_{h, n_{1}, \ldots, n_{k}}$, we associate with a unique $n$-square matrix, denoted $A(\alpha)$, to be $A(\alpha):=A$ when $n=1$, and

(3) $\quad A(\alpha):=\sum_{\substack{1 \leqslant s<h \\ s \text { odd }}} A_{\alpha_{s}}+\sum_{\substack{1 \leqslant s<h \\ s \text { even }}} A_{\alpha_{s}}^{t}-\sum_{1<s<h} a_{\alpha_{s} \alpha_{s}} E_{\alpha_{s} \alpha_{s}}$ when $n \geqslant 2$

where, for each $1 \leqslant s<h, A_{\alpha_{s}}=\left(b_{i j}\right)$ is the $n$-square matrix whose entries are given by

$$
b_{i j}=\left\{\begin{array}{cc}
a_{i j} & \text { if } \quad \alpha_{s} \leqslant i, j \leqslant \alpha_{s+1} \\
0 & \text { otherwise. }
\end{array}\right.
$$

Furthermore, if $\sigma_{1}, \ldots, \sigma_{h-1}$ are field homomorphisms on $\mathbb{F}$, we use the notation $A(\alpha)^{\sigma_{1} \ldots \sigma_{h-1}}$ to designate the $n$-square matrix defined by

$$
A(\alpha)^{\sigma_{1} \ldots \sigma_{h-1}}:=\sum_{\substack{1 \leqslant s<h \\ s \text { odd }}} A_{\alpha_{s}}^{\sigma_{s}}+\sum_{\substack{1 \leqslant s<h \\ s \text { even }}}\left(A_{\alpha_{s}}^{\sigma_{s}}\right)^{t}-\sum_{1<s<h} \sigma_{s}\left(a_{\alpha_{s} \alpha_{s}}\right) E_{\alpha_{s} \alpha_{s}}
$$

when $n \geqslant 2$, and $A(\alpha)^{\sigma_{1} \ldots \sigma_{h-1}}:=A^{\sigma_{1}}$ when $n=1$. If $\sigma_{1}=\cdots=\sigma_{h-1}=$ $\sigma$, then $A(\alpha)^{\sigma_{1} \ldots \sigma_{h-1}}=A(\alpha)^{\sigma}$. For example, if $A=\left(a_{i j}\right) \in \mathcal{T}_{6}(\mathbb{F}), \alpha=$ $(1,2,3,6) \in H_{4,6}$, and $\sigma_{1}, \sigma_{2}, \sigma_{3}$ are field homomorphisms on $\mathbb{F}$, then

$$
A(\alpha)^{\sigma_{1}, \sigma_{2}, \sigma_{3}}=\left(\begin{array}{cccccc}
\sigma_{1}\left(a_{11}\right) & \sigma_{1}\left(a_{12}\right) & 0 & 0 & 0 & 0 \\
0 & \sigma_{1}\left(a_{22}\right) & 0 & 0 & 0 & 0 \\
0 & \sigma_{2}\left(a_{23}\right) & \sigma_{2}\left(a_{33}\right) & \sigma_{3}\left(a_{34}\right) & \sigma_{3}\left(a_{35}\right) & \sigma_{3}\left(a_{36}\right) \\
0 & 0 & 0 & \sigma_{3}\left(a_{44}\right) & \sigma_{3}\left(a_{45}\right) & \sigma_{3}\left(a_{46}\right) \\
0 & 0 & 0 & 0 & \sigma_{3}\left(a_{55}\right) & \sigma_{3}\left(a_{56}\right) \\
0 & 0 & 0 & 0 & 0 & \sigma_{3}\left(a_{66}\right)
\end{array}\right) .
$$

Let $m, n$ and $p$ be integers with $1 \leqslant n \leqslant m$ and $0 \leqslant p \leqslant m-n$. We recall that $E_{i j}$ and $F_{i j}$ denote the matrix units in $\mathcal{M}_{n}(\mathbb{F})$ and $\mathcal{M}_{m}(\mathbb{F})$, respectively. Let $\Phi: \mathcal{T}_{n_{1}, \ldots, n_{k}}(\mathbb{F}) \rightarrow \mathcal{M}_{m}(\mathbb{F})$ be the additive map defined by

- $\Phi(A)=\left(\begin{array}{ccc}0_{p} & 0 & 0 \\ 0 & A(\alpha)^{\sigma_{1} \ldots \sigma_{h-1}} & 0 \\ 0 & 0 & 0\end{array}\right)$ for all $A=\left(a_{i j}\right)-\sum_{s=1}^{h} a_{\alpha_{s} \alpha_{s}} E_{\alpha_{s} \alpha_{s}}$ $\in \mathcal{T}_{n_{1}, \ldots, n_{k}}(\mathbb{F})$,

(I) $-\Phi\left(a E_{\alpha_{s} \alpha_{s}}\right)=\sum_{j=1}^{m} g_{s j}(a) F_{p+\alpha_{s}, j}$ for all $a \in \mathbb{F}$, whenever $1 \leqslant s \leqslant h$ is odd, and

- $\Phi\left(a E_{\alpha_{s} \alpha_{s}}\right)=\sum_{j=1}^{m} g_{s j}(a) F_{j, p+\alpha_{s}}$ for all $a \in \mathbb{F}$, whenever $1 \leqslant s \leqslant h$ is even,

where $\alpha=\left(\alpha_{1}, \ldots, \alpha_{h}\right) \in H_{h, n_{1}, \ldots, n_{k}}, \sigma_{1}, \ldots, \sigma_{h-1}$ are nonzero field homomorphisms on $\mathbb{F}$, and $g_{11}, \ldots, g_{h m}$ are additive maps on $\mathbb{F}$ such that 
(i) for each $1 \leqslant s \leqslant h, g_{s, p+\alpha_{s}}(1)=1$ and $g_{s j}(1)=0$ for $j \neq p+\alpha_{s}$,

(ii) when $n_{1} \geqslant 2, g_{1, p+1}=\sigma_{1}$ and $g_{1 j}=0$ for every $j \neq p+1$, and

(iii) when $n_{k} \geqslant 2, g_{h, p+n}=\sigma_{h-1}$ and $g_{h j}=0$ for every $j \neq p+n$.

In view of Definitions (3) and (4), it is easy to check that $\operatorname{rank} A(\alpha)^{\sigma_{1} \ldots \sigma_{h-1}} \leqslant 1$ whenever $A$ is of rank one. Consequently, the map $\Phi$ defined in (I) is a rank-one nonincreasing additive map with $\Phi\left(I_{n}\right)=0_{p} \oplus I_{n} \oplus 0_{m-p-n}$.

An additive map $\psi: \mathcal{T}_{n_{1}, \ldots, n_{k}}(\mathbb{F}) \rightarrow \mathcal{M}_{m}(\mathbb{F})$ is said to be $(n, p)$-block rankone nonincreasing if $\psi$ or $\psi^{t}$ is of the form $\Phi$ defined in $(\mathbf{I})$. Here, $\psi^{t}$ is the map defined by $\psi^{t}(A)=\psi(A)^{t}$ for all $A \in \mathcal{T}_{n_{1}, \ldots, n_{k}}(\mathbb{F})$.

We state the following result proved in [3, Theorem 2.10].

Lemma 2.6. Let $\mathbb{F}$ be a field, and let $m$ and $n$ be positive integers with $m \geqslant$ $n \geqslant 1$. Then $\psi: \mathcal{T}_{n_{1}, \ldots, n_{k}}(\mathbb{F}) \rightarrow \mathcal{M}_{m}(\mathbb{F})$ is a rank-one nonincreasing additive map with $\psi\left(I_{n}\right)$ being of rank $n$ if and only if there exist invertible matrices $P, Q \in \mathcal{M}_{m}(\mathbb{F})$ and integers $0=s_{0}<s_{1}<\cdots<s_{r}=k$ such that

$$
\psi(A)=P\left(\sum_{i=1}^{r} \Phi_{i}\left(A_{i}\right)\right) Q \quad \text { for all } A \in \mathcal{T}_{n_{1}, \ldots, n_{k}}(\mathbb{F}) .
$$

Here, $\bigoplus_{i=1}^{r} A_{i}$ is the $\left(\epsilon_{1}, \ldots, \epsilon_{r}\right)$-block diagonal matrix induced by $A$ with $\epsilon_{i}=$ $\delta_{s_{i}}-\delta_{s_{i-1}}, \delta_{s_{i}}=n_{1}+\cdots+n_{s_{i}}$ and $\delta_{0}=0$, and for each $1 \leqslant i \leqslant r$, $\Phi_{i}: \mathcal{T}_{n_{s_{i-1}+1}, \ldots, n_{s_{i}}}(\mathbb{F}) \rightarrow \mathcal{M}_{m}(\mathbb{F})$ is a $\left(\epsilon_{i}, \delta_{s_{i-1}}\right)$-block rank-one nonincreasing additive map as described in (I).

Lemma 2.7. Let $\mathbb{F}$ be a field. Let $r, \epsilon_{1}, \ldots, \epsilon_{r}$ be positive integers such that $\epsilon_{1}+\cdots+\epsilon_{r}=n$. Let $A \in \mathcal{T}_{n}(\mathbb{F})$, and let $\alpha_{i} \in H_{k_{i}, \epsilon_{i}}$ with positive integer $k_{i} \leqslant \epsilon_{i}$ for $i=1, \ldots, r$. Then

$$
C_{n-1}\left(\bigoplus_{i=1}^{r} A_{i}\left(\alpha_{i}\right)\right)=\left(\bigoplus_{i=1}^{r} C_{n-1}\left(A^{\sim}\right)_{i}\left(\alpha_{i}\right)\right)^{\sim}
$$

where $\bigoplus_{i=1}^{r} A_{i}$ and $\bigoplus_{i=1}^{r} C_{n-1}\left(A^{\sim}\right)_{i}$ are the $\left(\epsilon_{1}, \ldots, \epsilon_{r}\right)$-block diagonal matrices induced by $A$ and $C_{n-1}\left(A^{\sim}\right)$, respectively.

Proof. Let $N=\{1, \ldots, n\}$ and let

$$
\mathscr{I}=\left\{(s, t) \in N \times N \mid E_{s t} \in \mathcal{T}_{n}(\mathbb{F}) \text { and } \bigoplus_{i=1}^{r}\left(E_{s t}\right)_{i} \neq 0\right\},
$$

where $\bigoplus_{i=1}^{r}\left(E_{s t}\right)_{i}$ is the $\left(\epsilon_{1}, \ldots, \epsilon_{r}\right)$-block diagonal matrix induced by $E_{s t}$. Let $A \in \mathcal{T}_{n}(\mathbb{F})$. We denote

$$
X=\left(x_{s t}\right)=\operatorname{adj}\left(\bigoplus_{i=1}^{r} A_{i}\left(\alpha_{i}\right)\right) \quad \text { and } \quad Y=\left(y_{s t}\right)=\bigoplus_{i=1}^{r}(\operatorname{adj} A)_{i}\left(\alpha_{i}\right),
$$

where $\bigoplus_{i=1}^{r} A_{i}$ and $\bigoplus_{i=1}^{r}(\operatorname{adj} A)_{i}$ are the $\left(\epsilon_{1}, \ldots, \epsilon_{r}\right)$-block diagonal matrices induced by $A$ and $\operatorname{adj} A$, respectively. We first claim that $X=Y$. Since the classical adjoint of a block triangular matrix is also a block triangular matrix 
of the same type, it follows that $x_{s t}=0=y_{s t}$ for every $(s, t) \notin \mathscr{I}$. We now consider $x_{p q}$ with $(p, q) \in \mathscr{I}$. Then there exists $1 \leqslant i_{0} \leqslant r$ such that $\left(E_{p q}\right)_{i_{0}} \neq 0$. So,

$$
\begin{aligned}
x_{p q} & =(-1)^{p+q} \operatorname{det}\left(\left(\bigoplus_{i=1}^{r} A_{i}\left(\alpha_{i}\right)\right)[q \mid p]\right) \\
& =(-1)^{p+q}\left(\prod_{i=1, i \neq i_{0}}^{r} \operatorname{det} A_{i}\left(\alpha_{i}\right)\right) \times \operatorname{det}\left(A_{i_{0}}\left(\alpha_{i_{0}}\right)[q \mid p]\right) .
\end{aligned}
$$

Since each $A_{i}$ is upper triangular, by Definition (3), we have $\operatorname{det} A_{i}\left(\alpha_{i}\right)=$ $\operatorname{det} A_{i}$, and so

$$
x_{p q}=(-1)^{p+q}\left(\prod_{i=1, i \neq i_{0}}^{r} \operatorname{det} A_{i}\right) \times \operatorname{det}\left(A_{i_{0}}\left(\alpha_{i_{0}}\right)[q \mid p]\right) .
$$

We now consider the following two cases. If $\left(E_{p q}\right)_{i_{0}}\left(\alpha_{i_{0}}\right)=0$, then $y_{p q}=0$ since $\operatorname{adj} A \in \mathcal{T}_{n}(\mathbb{F})$. On the other hand, it can be verified that $\operatorname{det}\left(A_{i_{0}}\left(\alpha_{i_{0}}\right)[q \mid p]\right)=0$, and so, $x_{p q}=0$ by (5). Hence, $x_{p q}=0=y_{p q}$. Next, we consider $\left(E_{s t}\right)_{i_{0}}\left(\alpha_{i_{0}}\right) \neq$ 0 . Since

$$
\operatorname{det}\left(A_{i_{0}}\left(\alpha_{i_{0}}\right)[q \mid p]\right)=\left\{\begin{array}{lll}
\operatorname{det}\left(A_{i_{0}}[q \mid p]\right) & \text { if } & \left(E_{p q}\right)_{i_{0}}\left(\alpha_{i_{0}}\right)=\left(E_{p q}\right)_{i_{0}} \\
\operatorname{det}\left(A_{i_{0}}[p \mid q]\right) & \text { if } & \left(E_{p q}\right)_{i_{0}}\left(\alpha_{i_{0}}\right)=\left(E_{p q}\right)_{i_{0}}^{t}=\left(E_{q p}\right)_{i_{0}} .
\end{array}\right.
$$

By (5),

$$
\begin{aligned}
x_{p q} & =\left\{\begin{array}{lll}
(-1)^{p+q}\left(\prod_{i=1, i \neq i_{0}}^{r} \operatorname{det} A_{i}\right) \times \operatorname{det}\left(A_{i_{0}}[q \mid p]\right) & \text { if } & \left(E_{p q}\right)_{i_{0}}\left(\alpha_{i_{0}}\right)=\left(E_{p q}\right)_{i_{0}} \\
(-1)^{p+q}\left(\prod_{i=1, i \neq i_{0}}^{r} \operatorname{det} A_{i}\right) \times \operatorname{det}\left(A_{i_{0}}[p \mid q]\right) & \text { if } & \left(E_{p q}\right)_{i_{0}}\left(\alpha_{i_{0}}\right)=\left(E_{q p}\right)_{i_{0}},
\end{array}\right. \\
& =\left\{\begin{array}{lll}
(-1)^{p+q} \operatorname{det}(A[q \mid p]) & \text { if } \quad\left(E_{p q}\right)_{i_{0}}\left(\alpha_{i_{0}}\right)=\left(E_{p q}\right)_{i_{0}} \\
(-1)^{p+q} \operatorname{det}(A[p \mid q]) & \text { if } & \left(E_{p q}\right)_{i_{0}}\left(\alpha_{i_{0}}\right)=\left(E_{q p}\right)_{i_{0}},
\end{array}\right. \\
& =\left\{\begin{array}{lll}
(\operatorname{adj} A)_{p q} & \text { if } & \left(E_{p q}\right)_{i_{0}}\left(\alpha_{i_{0}}\right)=\left(E_{p q}\right)_{i_{0}} \\
(\operatorname{adj} A)_{q p} & \text { if } & \left(E_{p q}\right)_{i_{0}}\left(\alpha_{i_{0}}\right)=\left(E_{q p}\right)_{i_{0}},
\end{array}\right. \\
& =y_{p q}
\end{aligned}
$$

since $\operatorname{adj} A \in \mathcal{T}_{n}(\mathbb{F})$. Hence, $x_{s t}=y_{s t}$ for every $1 \leqslant s, t \leqslant n$, as claimed. So, we have

$$
\operatorname{adj}\left(\bigoplus_{i=1}^{r} A_{i}\left(\alpha_{i}\right)\right)=\bigoplus_{i=1}^{r}(\operatorname{adj} A)_{i}\left(\alpha_{i}\right) .
$$

Since $C_{n-1}(A)^{\sim}=Z_{n}(\operatorname{adj} A) Z_{n}$, and $Z_{n}=\bigoplus_{i=1}^{r}\left(Z_{n}\right)_{i}$ and $\left(Z_{n}\right)_{i} A_{i}\left(\alpha_{i}\right)\left(Z_{n}\right)_{i}=$ $\left(Z_{n} A Z_{n}\right)_{i}\left(\alpha_{i}\right)$, where $\bigoplus_{i=1}^{r}\left(Z_{n}\right)_{i}$ and $\bigoplus_{i=1}^{r}\left(Z_{n} A Z_{n}\right)_{i}$ are the $\left(\epsilon_{1}, \ldots, \epsilon_{r}\right)$-block 
diagonal matrices induced by $Z_{n}$ and $Z_{n} A Z_{n}$, respectively, and together with (6) and Lemma 2.3(xi), we obtain

$$
\begin{aligned}
\left(C_{n-1}\left(\bigoplus_{i=1}^{r} A_{i}\left(\alpha_{i}\right)\right)\right)^{\sim} & =Z_{n}\left(\operatorname{adj}\left(\bigoplus_{i=1}^{r} A_{i}\left(\alpha_{i}\right)\right)\right) Z_{n} \\
& =Z_{n}\left(\bigoplus_{i=1}^{r}(\operatorname{adj} A)_{i}\left(\alpha_{i}\right)\right) Z_{n} \\
& =\bigoplus_{i=1}^{r}\left(Z_{n}\right)_{i}(\operatorname{adj} A)_{i}\left(\alpha_{i}\right)\left(Z_{n}\right)_{i} \\
& =\bigoplus_{i=1}^{r} C_{n-1}\left(A^{\sim}\right)_{i}\left(\alpha_{i}\right) .
\end{aligned}
$$

Here, $\bigoplus_{i=1}^{r} C_{n-1}\left(A^{\sim}\right)_{i}$ is the $\left(\epsilon_{1}, \ldots, \epsilon_{r}\right)$-block diagonal matrix induced by $C_{n-1}\left(A^{\sim}\right)$. This completes our proof.

By Lemmas 2.5, 2.6 and 2.7, we give a general form of compond-commuting additive maps from triangular matrices to square matrices over an arbitrary field. More precisely, we have:

Theorem 2.8. Let $\mathbb{F}$ be a field, and let $m$ and $n$ be integers with $m, n \geqslant 3$. Then $\psi: \mathcal{T}_{n}(\mathbb{F}) \rightarrow \mathcal{M}_{m}(\mathbb{F})$ is a compound-commuting additive map if and only if either $\psi=0$, or $m=n$ and there exist positive integers $0=s_{0}<s_{1}<\cdots<$ $s_{r}=n$ with $s_{i}-s_{i-1}=s_{r+1-i}-s_{r-i}$ for $i=1, \ldots, r$, and a nonzero field homomorphism $\sigma: \mathbb{F} \rightarrow \mathbb{F}$ such that

$$
\psi(A)=P\left(\bigoplus_{i=1}^{r}\left[\lambda_{i} A_{i}\left(\alpha_{i}\right)+\left(1-\lambda_{i}\right) A_{i}\left(\alpha_{i}\right)^{t}\right]^{\sigma}\right) Q \quad \text { for all } A \in \mathcal{T}_{n}(\mathbb{F}) .
$$

Here, $\bigoplus_{i=1}^{r} A_{i}$ is the $\left(\epsilon_{1}, \ldots, \epsilon_{r}\right)$-block diagonal matrix induced by $A$ with $\epsilon_{i}=$ $s_{i}-s_{i-1}$ and $\epsilon_{i}=\epsilon_{r+1-i}$ for $i=1, \ldots, r ; \alpha_{i} \in H_{k_{i}, \epsilon_{i}}$ for some positive integer $k_{i} \leqslant \epsilon_{i}$ with $k_{i}=k_{r+1-i}$ and $\alpha_{i}^{\sim}=\alpha_{r+1-i}$ for $i=1, \ldots, r ; P, Q \in \mathcal{M}_{n}(\mathbb{F})$ are invertible with $C_{n-1}(P)=P\left(\bigoplus_{i=1}^{r} \mu_{i} I_{\epsilon_{i}}\right)$ and $C_{n-1}(Q)=\left(\bigoplus_{i=1}^{r} \mu_{i}^{-1} I_{\epsilon_{i}}\right) Q$ for some nonzero scalars $\mu_{1}, \ldots, \mu_{r} \in \mathbb{F}$; and $\lambda_{1}, \ldots, \lambda_{r} \in\{0,1\}$ with $\lambda_{r+1-i}=\lambda_{i}$ when $k_{i}$ even, or $\lambda_{r+1-i}=1-\lambda_{i}$ when $k_{i}$ odd for $i=1, \ldots, r$.

Proof. Sufficiency part. It is clear that $\psi=0$ is a compound-commuting additive map. Suppose $\psi \neq 0$ and it is of the form stated in Theorem 2.8. Let $A \in \mathcal{T}_{n}(\mathbb{F})$. By Lemma 2.7, it is easily checked that

$$
\begin{aligned}
& C_{n-1}\left(\bigoplus_{i=1}^{r} \lambda_{i} A_{i}\left(\alpha_{i}\right)+\left(1-\lambda_{i}\right) A_{i}\left(\alpha_{i}\right)^{t}\right) \\
= & \left(\bigoplus_{i=1}^{r} \lambda_{i} C_{n-1}\left(A^{\sim}\right)_{i}\left(\alpha_{i}\right)+\left(1-\lambda_{i}\right) C_{n-1}\left(A^{\sim}\right)_{i}\left(\alpha_{i}\right)^{t}\right)^{\sim},
\end{aligned}
$$


where $\bigoplus_{i=1}^{r} A_{i}$ and $\bigoplus_{i=1}^{r} C_{n-1}\left(A^{\sim}\right)_{i}$ are the $\left(\epsilon_{1}, \ldots, \epsilon_{r}\right)$-block diagonal matrices induced by $A$ and $C_{n-1}\left(A^{\sim}\right)$, respectively.

Since $\left(\bigoplus_{i=1}^{r} A_{i}\right)^{\sim}=\bigoplus_{i=1}^{r}\left(A_{r+1-i}\right)^{\sim}$ and

$$
A_{i}\left(\alpha_{i}\right)^{\sim}= \begin{cases}\left(A_{i}\right)^{\sim}\left(\alpha_{i}^{\sim}\right) & \text { when } \alpha_{i} \in H_{k_{i}, \epsilon_{i}} \text { with } k_{i} \text { even, } \\ \left(A_{i}\right)^{\sim}\left(\alpha_{i}^{\sim}\right)^{t} & \text { when } \alpha_{i} \in H_{k_{i}, \epsilon_{i}} \text { with } k_{i} \text { odd, }\end{cases}
$$

it follows that

$$
\begin{aligned}
& \left(\bigoplus_{i=1}^{r} \lambda_{i} C_{n-1}\left(A^{\sim}\right)_{i}\left(\alpha_{i}\right)+\left(1-\lambda_{i}\right) C_{n-1}\left(A^{\sim}\right)_{i}\left(\alpha_{i}\right)^{t}\right)^{\sim} \\
= & \bigoplus_{i=1}^{r}\left[\lambda_{r+1-i} C_{n-1}\left(A^{\sim}\right)_{r+1-i}\left(\alpha_{r+1-i}\right)+\left(1-\lambda_{r+1-i}\right) C_{n-1}\left(A^{\sim}\right)_{r+1-i}\left(\alpha_{r+1-i}\right)^{t}\right]^{\sim} \\
= & \bigoplus_{i=1}^{r} \beta_{r+1-i}\left(C_{n-1}\left(A^{\sim}\right)_{r+1-i}\right)^{\sim}\left(\alpha_{r+1-i}^{\sim}\right)+\left(1-\beta_{r+1-i}\right)\left(C_{n-1}\left(A^{\sim}\right)_{r+1-i}\right)^{\sim}\left(\alpha_{r+1-i}^{\sim}\right)^{t}
\end{aligned}
$$

where $\beta_{r+1-i}=\lambda_{r+1-i}$ when $k_{r+1-i}$ is even, or $\beta_{r+1-i}=1-\lambda_{r+1-i}$ when $k_{r+1-i}$ is odd for $i=1, \ldots, r$. Since, for each $1 \leqslant i \leqslant r$, we have $\lambda_{r+1-i}=\lambda_{i}$ when $k_{i}$ is even, or $\lambda_{r+1-i}=1-\lambda_{i}$ when $k_{i}$ is odd, it follows that $\beta_{r+1-i}=\lambda_{i}$ for $i=1, \ldots, r$. Together with the fact that $k_{r+1-i}=k_{i}$ and $\alpha_{r+1-i}^{\sim}=\alpha_{i}$ for $i=1, \ldots, r$, we obtain

$$
\begin{aligned}
& \bigoplus_{i=1}^{r} \beta_{r+1-i}\left(C_{n-1}\left(A^{\sim}\right)_{r+1-i}\right)^{\sim}\left(\alpha_{r+1-i}^{\sim}\right)+\left(1-\beta_{r+1-i}\right)\left(C_{n-1}\left(A^{\sim}\right)_{r+1-i}\right)^{\sim}\left(\alpha_{r+1-i}^{\sim}\right)^{t} \\
= & \bigoplus_{i=1}^{r} \lambda_{i}\left(C_{n-1}\left(A^{\sim}\right)_{r+1-i}\right)^{\sim}\left(\alpha_{i}\right)+\left(1-\lambda_{i}\right)\left(C_{n-1}\left(A^{\sim}\right)_{r+1-i}\right)^{\sim}\left(\alpha_{i}\right)^{t} .
\end{aligned}
$$

Further, since $\epsilon_{r+1-i}=\epsilon_{i}$ for $i=1, \ldots, r$, we have

$$
\bigoplus_{i=1}^{r}\left(A^{\sim}\right)_{i}=\bigoplus_{i=1}^{r}\left(A_{r+1-i}\right)^{\sim}
$$

and so,

$$
\bigoplus_{i=1}^{r}\left(A_{r+1-i}\right)^{\sim}\left(\alpha_{i}\right)=\bigoplus_{i=1}^{r}\left(A^{\sim}\right)_{i}\left(\alpha_{i}\right)
$$

where $\bigoplus_{i=1}^{r}\left(A^{\sim}\right)_{i}$ is the $\left(\epsilon_{1}, \ldots, \epsilon_{r}\right)$-block diagonal matrix induced by $A^{\sim}$. Together with the fact that $C_{n-1}(A)^{\sim}=C_{n-1}\left(A^{\sim}\right)$, we see that

$$
\begin{aligned}
& \bigoplus_{i=1}^{r} \lambda_{i}\left(C_{n-1}\left(A^{\sim}\right)_{r+1-i}\right)^{\sim}\left(\alpha_{i}\right)+\left(1-\lambda_{i}\right)\left(C_{n-1}\left(A^{\sim}\right)_{r+1-i}\right)^{\sim}\left(\alpha_{i}\right)^{t} \\
= & \bigoplus_{i=1}^{r} \lambda_{i}\left(C_{n-1}\left(A^{\sim}\right)^{\sim}\right)_{i}\left(\alpha_{i}\right)+\left(1-\lambda_{i}\right)\left(C_{n-1}\left(A^{\sim}\right)^{\sim}\right)_{i}\left(\alpha_{i}\right)^{t}
\end{aligned}
$$




$$
=\bigoplus_{i=1}^{r} \lambda_{i} C_{n-1}(A)_{i}\left(\alpha_{i}\right)+\left(1-\lambda_{i}\right) C_{n-1}(A)_{i}\left(\alpha_{i}\right)^{t} .
$$

Therefore,

$$
\begin{aligned}
C_{n-1}(\psi(A)) & =C_{n-1}\left(P\left(\bigoplus_{i=1}^{r}\left[\lambda_{i} A_{i}\left(\alpha_{i}\right)+\left(1-\lambda_{i}\right) A_{i}\left(\alpha_{i}\right)^{t}\right]^{\sigma}\right) Q\right) \\
& =C_{n-1}(P) C_{n-1}\left(\bigoplus_{i=1}^{r}\left[\lambda_{i} A_{i}\left(\alpha_{i}\right)+\left(1-\lambda_{i}\right) A_{i}\left(\alpha_{i}\right)^{t}\right]\right)^{\sigma} C_{n-1}(Q) \\
& =P\left(\bigoplus_{i=1}^{r} \lambda_{i} C_{n-1}(A)_{i}\left(\alpha_{i}\right)+\left(1-\lambda_{i}\right) C_{n-1}(A)_{i}\left(\alpha_{i}\right)^{t}\right)^{\sigma} Q \\
& =\psi\left(C_{n-1}(A)\right) .
\end{aligned}
$$

Hence, $\psi$ is a compound-commuting additive map.

Necessity part. If $\psi \neq 0$, then, by Lemma 2.5 , we have $m=n$, and $\psi$ is a rank-one nonincreasing additive map with $\psi\left(I_{n}\right)$ being of rank $n$. In view of Lemma 2.6, there exist integers $0=s_{0}<s_{1}<\cdots<s_{r}=n$, and invertible matrices $P, Q \in \mathcal{M}_{n}(\mathbb{F})$ such that

$$
\psi(A)=P\left(\sum_{i=1}^{r} \Phi_{i}\left(A_{i}\right)\right) Q \quad \text { for all } A \in \mathcal{T}_{n}(\mathbb{F}) .
$$

Here, $\bigoplus_{i=1}^{r} A_{i}$ is the $\left(\epsilon_{1}, \ldots, \epsilon_{r}\right)$-block diagonal matrix induced by $A$ with $\epsilon_{i}=s_{i}-s_{i-1}$ for $i=1, \ldots, r$, and each $\Phi_{i}: \mathcal{T}_{\epsilon_{i}}(\mathbb{F}) \rightarrow \mathcal{M}_{n}(\mathbb{F})$ is a $\left(\epsilon_{i}, s_{i-1}\right)$ block rank-one nonincreasing additive map as described in (I), i.e., either $\Phi_{i}$ or $\Phi_{i}^{t}$ is of the form:

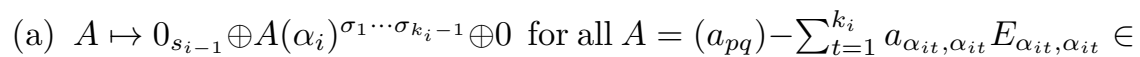
$\mathcal{T}_{\epsilon_{i}}(\mathbb{F})$,

(II) (b) $a E_{\alpha_{i t}, \alpha_{i t}} \mapsto \sum_{j=1}^{n} g_{t j}(a) E_{s_{i-1}+\alpha_{i t}, j}$ for all $a \in \mathbb{F}$, whenever $1 \leqslant t \leqslant k_{i}$ is odd, and

(c) $a E_{\alpha_{i t}, \alpha_{i t}} \mapsto \sum_{j=1}^{n} g_{t j}(a) E_{j, s_{i-1}+\alpha_{i t}}$ for all $a \in \mathbb{F}$, whenever $1 \leqslant t \leqslant k_{i}$ is even.

Here, $\sigma_{1}, \ldots, \sigma_{k_{i}-1}$ are nonzero field homomorphisms on $\mathbb{F}, \alpha_{i}=\left(\alpha_{i 1}, \ldots, \alpha_{i k_{i}}\right)$ $\in H_{k_{i}, \epsilon_{i}}$ for $i=1, \ldots, n$, and $g_{11}, \ldots, g_{k_{i}, n}$ are additive maps on $\mathbb{F}$ such that for each $1 \leqslant t \leqslant k_{i}, g_{t, s_{i-1}+\alpha_{i t}}(1)=1$ and $g_{t j}(1)=0$ for every $j \neq s_{i-1}+\alpha_{i t}$. Let $\varphi: \mathcal{T}_{n}(\mathbb{F}) \rightarrow \mathcal{M}_{n}(\mathbb{F})$ be the additive map defined by

$$
\varphi(A)=P^{-1} \psi(A) Q^{-1} \quad \text { for all } A \in \mathcal{T}_{n}(\mathbb{F}) .
$$

By (8) and (9), we get

$$
\varphi(A)=\sum_{i=1}^{r} \Phi_{i}\left(A_{i}\right) \quad \text { for all } A \in \mathcal{T}_{n}(\mathbb{F}) .
$$


By (8) and the structure of $\Phi_{i}$ 's as described in (II), we have $\psi\left(I_{n}\right)=P Q$. Since $C_{n-1}\left(\psi\left(I_{n}\right)\right)=\psi\left(C_{n-1}\left(I_{n}\right)\right)=\psi\left(I_{n}\right)$, we conclude that $C_{n-1}(P Q)=P Q$, and so, $P^{-1} C_{n-1}(P)=Q C_{n-1}\left(Q^{-1}\right)$. Let $1 \leqslant j \leqslant n$. Since $\psi\left(C_{n-1}\left(I_{n}-\right.\right.$ $\left.\left.E_{n+1-j, n+1-j}\right)\right)=C_{n-1}\left(\psi\left(I_{n}-E_{n+1-j, n+1-j}\right)\right)$, it follows from (8) and (II) that $P E_{j j} Q=C_{n-1}(P) E_{j j} C_{n-1}(Q)$. Thus

$P^{-1} C_{n-1}(P) E_{j j}=E_{j j} Q C_{n-1}\left(Q^{-1}\right)=E_{j j} P^{-1} C_{n-1}(P) \quad$ for all $j=1, \ldots, n$.

Therefore, $P^{-1} C_{n-1}(P)=D$ for some invertible diagonal matrix $D=\operatorname{diag}\left(d_{1}\right.$, $\left.\ldots, d_{n}\right) \in \mathcal{M}_{n}(\mathbb{F})$. Hence

$$
C_{n-1}(P)=P D \quad \text { and } \quad C_{n-1}(Q)=D^{-1} Q .
$$

Since $\psi\left(C_{n-1}(A)\right)=C_{n-1}(\psi(A))$ for all $A \in \mathcal{T}_{n}(\mathbb{F})$, it follows from (9) and (11) that

$$
\varphi\left(C_{n-1}(A)\right)=D C_{n-1}(\varphi(A)) D^{-1} \quad \text { for all } A \in \mathcal{T}_{n}(\mathbb{F}) .
$$

In view of (10), and by the structure of $\Phi_{i}$ 's as described in (II), we see that $\varphi\left(E_{s s}\right)=E_{s s}$ for all $s$, and when $s<t$, we have $\varphi\left(E_{s t}\right)=E_{s t}$ or $\varphi\left(E_{s t}\right)=E_{t s}$ for every $E_{s t} \notin \operatorname{ker} \varphi$. Here, $\operatorname{ker} \varphi$ stands for the kernel of $\varphi$. We now show that if $E_{s t} \notin \operatorname{ker} \varphi$, then

$$
\begin{aligned}
& \varphi\left(E_{s t}\right)=E_{s t} \quad \Rightarrow \quad \varphi\left(E_{n+1-t, n+1-s}\right)=E_{n+1-t, n+1-s} \\
& \varphi\left(E_{s t}\right)=E_{t s} \quad \Rightarrow \quad \varphi\left(E_{n+1-t, n+1-s}\right)=E_{n+1-s, n+1-t} .
\end{aligned}
$$

We show only (13) as (14) can be proved similarly. By Lemma 2.2(iii)(b) and (12), we have

$$
\begin{aligned}
\varphi\left(E_{n+1-t, n+1-s}\right) & =\varphi\left(C_{n-1}\left(I_{n}-E_{s s}-E_{t t}+(-1)^{s+t+1} E_{s t}\right)\right) \\
& =D C_{n-1}\left(\varphi\left(I_{n}-E_{s s}-E_{t t}+(-1)^{s+t+1} E_{s t}\right)\right) D^{-1} \\
& \left.=D C_{n-1}\left(I_{n}-E_{s s}-E_{t t}+(-1)^{s+t+1} E_{s t}\right)\right) D^{-1} \\
& =D\left(E_{n+1-t, n+1-s}\right) D^{-1} \\
& =\left(d_{n+1-t} d_{n+1-s}^{-1}\right) E_{n+1-t, n+1-s .}
\end{aligned}
$$

Thus, $\varphi\left(E_{n+1-t, n+1-s}\right) \neq 0$. On the other hand, in view of the structure of $\Phi_{i}$ 's as described in $(\mathbf{I I})$, we know that $\varphi\left(E_{n+1-t, n+1-s}\right)=E_{n+1-t, n+1-s}$ or $\varphi\left(E_{n+1-t, n+1-s}\right)=E_{n+1-s, n+1-t}$. So, we conclude that $\varphi\left(E_{n+1-t, n+1-s}\right)=$ $E_{n+1-t, n+1-s}$, and thus, $\left(d_{n+1-t} d_{n+1-s}^{-1}\right)=1$, that is, $d_{n+1-t}=d_{n+1-s}$. Consequently, (13) is proved. Moreover, we note that if $E_{n+1-t, n+1-s} \notin \operatorname{ker} \varphi$, then, by the structure of $\Phi_{i}$ 's as described in (II), there exists an integer $1 \leqslant i_{0} \leqslant r$ such that $s_{i_{0}-1}+1 \leqslant n+1-s, n+t-t \leqslant s_{i_{0}}$. Therefore, the result $d_{n+1-t}=d_{n+1-s}$ whenever $E_{n+1-t, n+1-s} \notin \operatorname{ker} \varphi$ leads us to the conclusion $d_{s_{i-1}+1}=\cdots=d_{s_{i}}$ for every $i=1, \ldots, r$. Hence, we obtain $D=\bigoplus_{i=1}^{r} \mu_{i} I_{\epsilon_{i}}$ for some nonzero scalars $\mu_{1}, \ldots, \mu_{r} \in \mathbb{F}$, and by (11), we get $C_{n-1}(P)=P\left(\bigoplus_{i=1}^{r} \mu_{i} I_{\epsilon_{i}}\right)$ and $C_{n-1}(Q)=\left(\bigoplus_{i=1}^{r} \mu_{i}^{-1} I_{\epsilon_{i}}\right) Q$. 
We claim that there exists a nonzero homomorphism $\sigma: \mathbb{F} \rightarrow \mathbb{F}$ such that either

$$
\varphi\left(a E_{j j}\right)=\sigma(a) E_{j j} \quad \text { for every } a \in \mathbb{F} \text { and } 1 \leqslant j \leqslant n .
$$

By the structure of $\Phi_{i}$ 's as described in (II), we see that, for each $1 \leqslant j \leqslant n$, $\varphi\left(a E_{j j}\right)$ is of the form as described in (a), (b) or (c). We distinguish our proof into the following two cases:

Case I: Suppose there is an integer $1 \leqslant j_{0} \leqslant n$ such that $\varphi\left(a E_{j_{0}, j_{0}}\right)$ is of the form as described in (a), i.e., there exists a nonzero homomorphism $\sigma_{j_{0}}: \mathbb{F} \rightarrow \mathbb{F}$ such that $\varphi\left(a E_{j_{0}, j_{0}}\right)=\sigma_{j_{0}}(a) E_{j_{0}, j_{0}}$ for every $a \in \mathbb{F}$. Let $1 \leqslant j \neq j_{0} \leqslant n$ and $a \in \mathbb{F}$. If $j \neq n+1-j_{0}$, then, by Lemma 2.2(iii)(a) and (12), we have

$$
\begin{aligned}
\varphi\left(a E_{j j}\right) & =\varphi\left(C_{n-1}\left(I_{n}-E_{n+1-j, n+1-j}+(a-1) E_{j_{0}, j_{0}}\right)\right) \\
& =D C_{n-1}\left(\varphi\left(I_{n}-E_{n+1-j, n+1-j}+(a-1) E_{j_{0}, j_{0}}\right)\right) D^{-1} \\
& =D C_{n-1}\left(I_{n}-E_{n+1-j, n+1-j}+\left(\sigma_{j_{0}}(a)-1\right) E_{j_{0}, j_{0}}\right) D^{-1} \\
& =D\left(\sigma_{j_{0}}(a) E_{j j}\right) D^{-1}=\sigma_{j_{0}}(a) E_{j j} .
\end{aligned}
$$

If $j=n+1-j_{0}$, then, since $n \geqslant 3$, we select $1 \leqslant j_{1} \leqslant n$ with $j_{1} \neq j_{0}, n+1-j_{0}$, and so

$$
\begin{aligned}
\varphi\left(a E_{n+1-j_{0}, n+1-j_{0}}\right) & =\varphi\left(C_{n-1}\left(I_{n}-E_{j_{0}, j_{0}}+(a-1) E_{j_{1}, j_{1}}\right)\right) \\
& =D C_{n-1}\left(I_{n}-E_{j_{0}, j_{0}}+\left(\sigma_{j_{0}}(a)-1\right) E_{j_{1}, j_{1}}\right) D^{-1} \\
& =\sigma_{j_{0}}(a) E_{n+1-j_{0}, n+1-j_{0}} .
\end{aligned}
$$

Consequently, we conclude that there exists a nonzero homomorphism $\sigma: \mathbb{F} \rightarrow$ $\mathbb{F}$ such that $\varphi\left(a E_{j j}\right)=\sigma(a) E_{j j}$ for all $1 \leqslant j \leqslant n$ and $a \in \mathbb{F}$. We are done.

Case II: Suppose, for each $1 \leqslant j \leqslant n, \varphi\left(a E_{j j}\right)$ is of the form as described in (b) or (c) only. We divide our argument into two cases:

Subcase 1: Suppose there exist integers $1 \leqslant s_{1} \neq s_{2} \leqslant n$ such that $\psi\left(a E_{s_{1}, s_{1}}\right)$ and $\varphi\left(a E_{s_{2}, s_{2}}\right)$ are of distinct forms. We may assume, without loss of generality, that $\psi\left(a E_{s_{1}, s_{1}}\right)$ and $\varphi\left(a E_{s_{2}, s_{2}}\right)$ are of the forms as described in (c) and (b), respectively, and $s_{2} \neq n+1-s_{1}$. Let $\varphi\left(a E_{s_{1}, s_{1}}\right)=\sum_{j=1}^{n} g_{s_{1}, j}(a) E_{j, s_{1}}$ and $\varphi\left(a E_{s_{2}, s_{2}}\right)=\sum_{j=1}^{n} g_{s_{2}, j}(a) E_{s_{2}, j}$, where $g_{s_{1}, 1} \ldots, g_{s_{1}, n}, g_{s_{2}, 1} \ldots, g_{s_{2}, n}$, are additive maps on $\mathbb{F}$ such that $g_{s_{i}, s_{i}}(1)=1$ for $i=1,2$, and for each $i=1,2$, $g_{s_{i}, j}(1)=0$ for all $j \neq s_{i}$. By $(12)$, we see that

$$
\begin{aligned}
\varphi\left(a E_{s_{1}, s_{1}}\right) & =\varphi\left(C_{n-1}\left(I_{n}-E_{n+1-s_{1}, n+1-s_{1}}+(a-1) E_{s_{2}, s_{2}}\right)\right) \\
& =D C_{n-1}\left(\varphi\left(I_{n}-E_{n+1-s_{1}, n+1-s_{1}}-E_{s_{2}, s_{2}}+a E_{s_{2}, s_{2}}\right)\right) D^{-1} \\
& =D C_{n-1}\left(I_{n}-E_{n+1-s_{1}, n+1-s_{1}}-E_{s_{2}, s_{2}}+\sum_{j=1}^{n} g_{s_{2}, j}(a) E_{s_{2}, j}\right) D^{-1} \\
& =D\left(g_{s_{2}, s_{2}}(a) E_{s_{1}, s_{1}}+(-1)^{s_{1}+s_{2}+1} g_{s_{2}, n+1-s_{1}}(a) E_{s_{1}, n+1-s_{2}}\right) D^{-1} \\
& =g_{s_{2}, s_{2}}(a) E_{s_{1}, s_{1}}+(-1)^{s_{1}+s_{2}+1} g_{s_{2}, n+1-s_{1}}(a) \beta_{1} E_{s_{1}, n+1-s_{2}},
\end{aligned}
$$


where $D E_{s_{1}, n+1-s_{2}} D^{-1}=\beta_{1} E_{s_{1}, n+1-s_{2}}$ for some nonzero scalar $\beta_{1} \in \mathbb{F}$. Then

$$
\begin{aligned}
\sum_{j=1}^{n} g_{s_{1}, j}(a) E_{j, s_{1}} & =\varphi\left(a E_{s_{1}, s_{1}}\right) \\
& =g_{s_{2}, s_{2}}(a) E_{s_{1}, s_{1}}+(-1)^{s_{1}+s_{2}+1} g_{s_{2}, n+1-s_{1}}(a) \beta_{1} E_{s_{1}, n+1-s_{2}}
\end{aligned}
$$

and so, $g_{s_{1}, j}=0$ for all $j \neq s_{1}$. Hence, we obtain $\varphi\left(a E_{s_{1}, s_{1}}\right)=g_{s_{1}, s_{1}}(a) E_{s_{1}, s_{1}}$ for all $a \in \mathbb{F}$.

Subcase 2: Suppose all $\varphi\left(a E_{j j}\right)$ 's are of the same form. Without loss of generality, we assume $\varphi\left(a E_{j j}\right)$ is of form (b) for all $j=1, \ldots, n$. Since $n \geqslant 3$, there exist integers $1 \leqslant s_{1}<s_{2}<s_{3} \leqslant n$ such that $s_{2} \neq n+1-s_{1}$ and $s_{3} \neq n+1-s_{2}$. Let $\varphi\left(a E_{s_{i}, s_{i}}\right)=\sum_{j=1}^{n} g_{s_{i}, j}(a) E_{s_{i}, j}$ for $i=1,2,3$, where $g_{s_{1}, 1}, \ldots, g_{s_{1}, n}, g_{s_{2}, 1}, \ldots, g_{s_{3}, n}$ are additive maps on $\mathbb{F}$ with $g_{s_{i}, s_{i}}(1)=1$ for $i=$ $1,2,3$, and for each $1 \leqslant i \leqslant 3, g_{s_{i}, j}(1)=0$ for all $j \neq s_{i}$. By a similar argument as in Subcase 1 , we see that $\varphi\left(a E_{s_{2}, s_{2}}\right)=\varphi\left(C_{n-1}\left(I_{n}-E_{n+1-s_{2}, n+1-s_{2}}+(a-\right.\right.$ 1) $\left.E_{s_{3}, s_{3}}\right)$ ) leads to

$$
\begin{aligned}
\sum_{j=1}^{n} g_{s_{2}, j}(a) E_{s_{2}, j} & =\varphi\left(a E_{s_{2}, s_{2}}\right) \\
& =g_{s_{3}, s_{3}}(a) E_{s_{2}, s_{2}}+(-1)^{s_{2}+s_{3}+1} g_{s_{3}, n+1-s_{2}}(a) \beta_{2} E_{s_{2}, n+1-s_{3}}
\end{aligned}
$$

for some nonzero scalar $\beta_{2} \in \mathbb{F}$. So, $g_{s_{2}, j}=0$ for all $j \neq s_{2}, n+1-s_{3}$, and hence,

$$
\varphi\left(a E_{s_{2}, s_{2}}\right)=g_{s_{2}, s_{2}}(a) E_{s_{2}, s_{2}}+g_{s_{2}, n+1-s_{3}}(a) E_{s_{2}, n+1-s_{3}} .
$$

Next, we consider $\varphi\left(a E_{s_{1}, s_{1}}\right)=\varphi\left(C_{n-1}\left(I_{n}-E_{n+1-s_{1}, n+1-s_{1}}+(a-1) E_{s_{2}, s_{2}}\right)\right)$. By $(16)$, since $s_{1} \neq s_{3}$, we get $g_{s_{1}, j}=0$ for all $j \neq s_{1}$, and hence, $\varphi\left(a E_{s_{1}, s_{1}}\right)=$ $g_{s_{1}, s_{1}}(a) E_{s_{1}, s_{1}}$ for all $a \in \mathbb{F}$.

We denote $\sigma=g_{s_{1}, s_{1}}$ and $s_{1}=s$ for the sake of simplicity. In view of the proofs of Subcase 1 and Subcase 2, we have shown that there exist an integer $1 \leqslant s \leqslant n$ and an additive map $\sigma$ on $\mathbb{F}$ with $\sigma(1)=1$ such that $\varphi\left(a E_{s s}\right)=\sigma(a) E_{s s}$ for all $a \in \mathbb{F}$. Therefore, by repeating a similar argument as in the proof of Case $\mathbf{I}$, we can show that $\varphi\left(a E_{j j}\right)=\sigma(a) E_{j j}$ for all $1 \leqslant j \leqslant n$ and $a \in \mathbb{F}$. To complete the proof of Claim (15), it suffices to show that $\sigma$ is a field homomorphism on $\mathbb{F}$. Let $a, b \in \mathbb{F}$ and let $1 \leqslant t_{1} \neq t_{2} \leqslant n$ with $t_{i} \neq n+1-s$ for $i=1,2$. We see that $a b E_{s s}=C_{n-1}\left(I_{n}-E_{n+1-s, n+1-s}+\right.$ $\left.(a-1) E_{t_{1}, t_{1}}+(b-1) E_{t_{2}, t_{2}}\right)$. Thus

$$
\begin{aligned}
\sigma(a b) E_{s s} & =\varphi\left(a b E_{s s}\right) \\
& =C_{n-1}\left(\varphi\left(I_{n}-E_{n+1-s, n+1-s}+(a-1) E_{t_{1}, t_{1}}+(b-1) E_{t_{2}, t_{2}}\right)\right) \\
& =C_{n-1}\left(I_{n}-E_{n+1-s, n+1-s}+(\sigma(a)-1) E_{t_{1}, t_{1}}+(\sigma(b)-1) E_{t_{2}, t_{2}}\right) \\
& =\sigma(a) \sigma(b) E_{s s} .
\end{aligned}
$$

Hence $\sigma(a b)=\sigma(a) \sigma(b)$ for all $a, b \in \mathbb{F}$, so $\sigma$ is a homomorphism on $\mathbb{F}$. Thus (15) as claimed. 
We next claim that $\varphi\left(a E_{s t}\right)=\sigma(a) E_{s t}$ or $\varphi\left(a E_{s t}\right)=\sigma(a) E_{t s}$ for all $E_{s t} \in$ $\mathcal{T}_{n}(\mathbb{F}) \backslash \operatorname{ker} \varphi$ and $a \in \mathbb{F}$. Suppose there exists an integer $1 \leqslant j_{1} \leqslant n-1$ such that $E_{j_{1}, j_{1}+1} \notin \operatorname{ker} \varphi$. By the structure of $\Phi_{i}$ 's as described in (II)-(a), there exist an integer $1 \leqslant i_{1} \leqslant r$ with $s_{i_{1-1}+1} \leqslant j_{1} \leqslant s_{i_{1}}$, and a nonzero homomorphism $\sigma_{j_{1}}: \mathbb{F} \rightarrow \mathbb{F}$ such that $\varphi\left(a E_{j_{1}, j_{1}+1}\right)=\sigma_{j_{1}}(a) E_{j_{1}, j_{1}+1}$ for all $a \in \mathbb{F}$, or $\varphi\left(a E_{j_{1}, j_{1}+1}\right)=\sigma_{j_{1}}(a) E_{j_{1}+1, j_{1}}$ for all $a \in \mathbb{F}$. We consider only the first case as the second case can be treated similarly. We first note, by (13), that $\varphi\left(E_{n-j_{1}, n+1-j_{1}}\right)=E_{n-j_{1}, n+1-j_{1}}$. Since $n \geqslant 3$, by choosing $1 \leqslant j \leqslant n$ with $j \neq j_{1}, j_{1}+1$, and together with (12) and (15), we have

$$
\begin{aligned}
& \varphi\left(a E_{j_{1}, j_{1}+1}\right) \\
= & \varphi\left(C_{n-1}\left(I_{n}-E_{n+1-j_{1}, n+1-j_{1}}-E_{n-j_{1}, n-j_{1}}+E_{n-j_{1}, n+1-j_{1}}+(a-1) E_{n+1-j, n+1-j}\right)\right) \\
= & D C_{n-1}\left(I_{n}-E_{n+1-j_{1}, n+1-j_{1}}-E_{n-j_{1}, n-j_{1}}+E_{n-j_{i}, n+1-j_{1}}+(\sigma(a)-1) E_{n+1-j, n+1-j}\right) D^{-1} \\
= & D\left(\sigma(a) E_{j_{1}, j_{1}+1}\right) D^{-1} .
\end{aligned}
$$

Since $s_{i_{1-1}+1} \leqslant j_{1} \leqslant s_{i_{1}}$ and $D=\bigoplus_{i=1}^{r} \mu_{i} I_{\epsilon_{i}}$, we get $D\left(\sigma(a) E_{j_{1}, j_{1}+1}\right) D^{-1}=$ $\sigma(a) E_{j_{1}, j_{1}+1}$, and so, $\sigma_{j_{1}}(a) E_{j_{1}, j_{1}+1}=\varphi\left(a E_{j_{1}, j_{1}+1}\right)=\sigma(a) E_{j_{1}, j_{1}+1}$ for all $a \in$ $\mathbb{F}$. Hence, $\sigma_{j_{1}}=\sigma$. Consequently, by (15) and together with the structures of $\Phi_{i}$ 's as described in (II), the claim is proved.

In view of $(9),(10),(15)$ and by the structures of $\Phi_{i}$ 's as described in (II), we obtain

$$
\psi(A)=P\left(\bigoplus_{i=1}^{r}\left[\lambda_{i} A_{i}\left(\alpha_{i}\right)+\left(1-\lambda_{i}\right) A_{i}\left(\alpha_{i}\right)^{t}\right]^{\sigma}\right) Q \quad \text { for all } A \in \mathcal{T}_{n}(\mathbb{F}) .
$$

where $\alpha_{i} \in H_{k_{i}, \epsilon_{i}}$ and $\lambda_{i} \in\{0,1\}$ for $i=1, \ldots, r$, and $P, Q \in \mathcal{M}_{n}(\mathbb{F})$ are invertible matrices with $C_{n-1}(P)=P\left(\bigoplus_{i=1}^{r} \mu_{i} I_{\epsilon_{i}}\right)$ and $C_{n-1}(Q)=\left(\bigoplus_{i=1}^{r} \mu_{i}^{-1} I_{\epsilon_{i}}\right) Q$ for some nonzero scalars $\mu_{1}, \ldots, \mu_{r} \in \mathbb{F}$. By (9), (13) and (14), we conclude that

$$
E_{j, j+1} \notin \operatorname{ker} \psi \quad \Leftrightarrow \quad E_{n-j, n+1-j} \notin \operatorname{ker} \psi \text {. }
$$

Thus, $\epsilon_{i}=\epsilon_{r+1-i}$ for every $i=1, \ldots, r$. Let $A \in \mathcal{T}_{n}(\mathbb{F})$. For each $1 \leqslant i \leqslant r$, we denote

$$
X_{i}=\left[\lambda_{i} A_{i}\left(\alpha_{i}\right)+\left(1-\lambda_{i}\right) A_{i}\left(\alpha_{i}\right)^{t}\right]^{\sigma}
$$

and

$$
Y_{i}=\left[\lambda_{i} C_{n-1}(A)_{i}\left(\alpha_{i}\right)+\left(1-\lambda_{i}\right) C_{n-1}(A)_{i}\left(\alpha_{i}\right)^{t}\right]^{\sigma},
$$

where $\bigoplus_{i=1}^{r} C_{n-1}(A)_{i}$ is the $\left(\epsilon_{1}, \ldots, \epsilon_{r}\right)$-block diagonal matrix induced by $C_{n-1}(A)$. Since $C_{n-1}(\psi(A))=\psi\left(C_{n-1}(A)\right)$, it follows that

$$
\begin{aligned}
C_{n-1}\left(\bigoplus_{i=1}^{r} X_{i}\right) & =C_{n-1}(P)^{-1} \psi\left(C_{n-1}(A)\right) C_{n-1}(Q)^{-1} \\
& =\left(\bigoplus_{i=1}^{r} \mu_{i}^{-1} I_{\epsilon_{i}}\right)\left(\bigoplus_{i=1}^{r} Y_{i}\right)\left(\bigoplus_{i=1}^{r} \mu_{i} I_{\epsilon_{i}}\right)
\end{aligned}
$$




$$
=\bigoplus_{i=1}^{r} Y_{i}
$$

Further since $\epsilon_{i}=\epsilon_{r+1-i}$ for all $i=1, \ldots, r$, we have

$$
\left(\bigoplus_{i=1}^{r} A_{i}\right)^{\sim}=\bigoplus_{i=1}^{r}\left(A_{r+1-i}\right)^{\sim}
$$

and together with (7) and Lemma 2.7, it is easy to see that

$$
\begin{aligned}
\bigoplus_{i=1}^{r} Y_{i} & =C_{n-1}\left(\bigoplus_{i=1}^{r} X_{i}\right) \\
& =C_{n-1}\left(\bigoplus_{i=1}^{r}\left[\lambda_{i} A_{i}\left(\alpha_{i}\right)+\left(1-\lambda_{i}\right) A_{i}\left(\alpha_{i}\right)^{t}\right]^{\sigma}\right) \\
& =\left(\left(\bigoplus_{i=1}^{r} \lambda_{i} C_{n-1}\left(A^{\sim}\right)_{i}\left(\alpha_{i}\right)+\left(1-\lambda_{i}\right) C_{n-1}\left(A^{\sim}\right)_{i}\left(\alpha_{i}\right)^{t}\right)^{\sim}\right)^{\sigma} \\
& =\bigoplus_{i=1}^{r}\left[\beta_{r+1-i} C_{n-1}(A)_{i}\left(\alpha_{r+1-i}^{\sim}\right)+\left(1-\beta_{r+1-i}\right) C_{n-1}(A)_{i}\left(\alpha_{r+1-i}^{\sim}\right)^{t}\right]^{\sigma},
\end{aligned}
$$

where $\beta_{r+1-i}=\lambda_{r+1-i}$ when $k_{r+1-i}$ is even, or $\beta_{r+1-i}=1-\lambda_{r+1-i}$ when $k_{r+1-i}$ is odd for $i=1, \ldots, r$. Thus, $Y_{i}=\left[\beta_{r+1-i} C_{n-1}(A)_{i}\left(\alpha_{r+1-i}^{\sim}\right)+(1-\right.$ $\left.\left.\beta_{r+1-i}\right) C_{n-1}(A)_{i}\left(\alpha_{r+1-i}^{\sim}\right)^{t}\right]^{\sigma}$ for all $i=1, \ldots, r$. Hence, for each $1 \leqslant i \leqslant r$, we have $\alpha_{i}^{\sim}=\alpha_{r+1-i}, k_{i}=k_{r+1-i}$, and $\lambda_{r+1-i}=\lambda_{i}$ when $k_{i}$ is even, or $\lambda_{r+1-i}=1-\lambda_{i}$ when $k_{i}$ is odd. This completes our proof.

We give two examples to illustrate the result obtained in Theorem 2.8.

Example 2.9. Let $\mathbb{F}$ be a field, and let $\sigma$ be a nonzero field homomorphism on $\mathbb{F}$. Let $\phi: \mathcal{T}_{5}(\mathbb{F}) \rightarrow \mathcal{M}_{5}(\mathbb{F})$ be the map defined by

$\phi(A)=\left(\begin{array}{ccccc}\sigma\left(a_{11}\right) & \sigma\left(a_{12}\right) & 0 & 0 & 0 \\ 0 & \sigma\left(a_{22}\right) & 0 & 0 & 0 \\ 0 & \sigma\left(a_{23}\right) & \sigma\left(a_{33}\right) & 0 & 0 \\ 0 & \sigma\left(a_{24}\right) & \sigma\left(a_{34}\right) & \sigma\left(a_{44}\right) & \sigma\left(a_{45}\right) \\ 0 & 0 & 0 & 0 & \sigma\left(a_{55}\right)\end{array}\right)$ for all $A=\left(a_{i j}\right) \in \mathcal{T}_{5}(\mathbb{F})$

We see that $\phi$ is a compound-commuting additive map and $\phi(A)^{\sim}=\phi\left(A^{\sim}\right)$ for all $A \in \mathcal{T}_{5}(\mathbb{F})$. 
Example 2.10. Let $\mathbb{F}$ be a field, and let $\sigma$ be a nonzero homomorphism on $\mathbb{F}$. Let $\psi: \mathcal{T}_{9}(\mathbb{F}) \rightarrow \mathcal{M}_{9}(\mathbb{F})$ be the map defined by

$$
\psi(A)=\left(\begin{array}{ccccccccc}
\sigma\left(a_{11}\right) & 0 & 0 & 0 & 0 & 0 & 0 & 0 & 0 \\
\sigma\left(a_{12}\right) & \sigma\left(a_{22}\right) & \sigma\left(a_{23}\right) & \sigma\left(a_{24}\right) & 0 & 0 & 0 & 0 & 0 \\
0 & 0 & \sigma\left(a_{33}\right) & \sigma\left(a_{34}\right) & 0 & 0 & 0 & 0 & 0 \\
0 & 0 & 0 & \sigma\left(a_{44}\right) & 0 & 0 & 0 & 0 & 0 \\
0 & 0 & 0 & 0 & \sigma\left(a_{55}\right) & 0 & 0 & 0 & 0 \\
0 & 0 & 0 & 0 & 0 & \sigma\left(a_{66}\right) & \sigma\left(a_{67}\right) & \sigma\left(a_{68}\right) & 0 \\
0 & 0 & 0 & 0 & 0 & 0 & \sigma\left(a_{77}\right) & \sigma\left(a_{78}\right) & 0 \\
0 & 0 & 0 & 0 & 0 & 0 & 0 & \sigma\left(a_{88}\right) & 0 \\
0 & 0 & 0 & 0 & 0 & 0 & 0 & \sigma\left(a_{89}\right) & \sigma\left(a_{99}\right)
\end{array}\right)
$$

for all $A=\left(a_{i j}\right) \in \mathcal{T}_{9}(\mathbb{F})$. It is easy to check that $\psi$ is a compound-commuting additive map and $\psi(A)^{\sim}=\psi\left(A^{\sim}\right)$ for all $A \in \mathcal{T}_{9}(\mathbb{F})$.

As an immediate consequence of Theorem 2.8, we have the following corollary.

Corollary 2.11. Let $\mathbb{F}$ be a field, and let $m$ and $n$ be integers with $m, n \geqslant 3$. Let $\psi: \mathcal{T}_{n}(\mathbb{F}) \rightarrow \mathcal{M}_{m}(\mathbb{F})$ be a compound-commuting additive map. Then the following statements are equivalent.

(a) $\psi$ is injective.

(b) $\psi\left(E_{1 n}\right) \neq 0$.

(c) $m=n$ and there exist a nonzero field homomorphism $\sigma: \mathbb{F} \rightarrow \mathbb{F}$ and invertible matrices $P, Q \in \mathcal{M}_{n}(\mathbb{F})$, with $C_{n-1}(P)=\mu P$ and $C_{n-1}(Q)=$ $\mu^{-1} Q$ for some nonzero element $\mu \in \mathbb{F}$, such that

$$
\begin{gathered}
\psi(A)=P A^{\sigma} Q \quad \text { for all } A \in \mathcal{M}_{n}(\mathbb{F}), \text { or } \\
\psi(A)=P\left(A^{\sigma}\right)^{t} Q \quad \text { for all } A \in \mathcal{M}_{n}(\mathbb{F}) .
\end{gathered}
$$

Proof. (a) $\Rightarrow$ (b) Trivial. (b) $\Rightarrow$ (c) Since $\psi$ is nonzero, it follows from Theorem 2.8 that $m=n$, and together with the fact that $\psi\left(E_{1 n}\right) \neq 0$, we conclude that there exist a scalar $\lambda \in\{0,1\}$, a nonzero field homomorphism $\sigma: \mathbb{F} \rightarrow \mathbb{F}$, and invertible matrices $P, Q \in \mathcal{M}_{n}(\mathbb{F})$, with $C_{n-1}(P)=\mu P$ and $C_{n-1}(Q)=\mu^{-1} Q$ for some nonzero element $\mu \in \mathbb{F}$, such that

$$
\psi(A)=P\left(\lambda A^{\sigma}+(1-\lambda)\left(A^{\sigma}\right)^{t}\right) Q \quad \text { for all } A \in \mathcal{M}_{n}(\mathbb{F}) .
$$

(c) $\Rightarrow$ (a) Trivial. We are done.

By Lemmas 2.5 and 2.6, we obtain a characterization of compound-commuting additive maps between square matrix algebras over the same field.

Theorem 2.12. Let $\mathbb{F}$ be a field, and let $m$ and $n$ be integers with $m, n \geqslant 3$. Then $\psi: \mathcal{M}_{n}(\mathbb{F}) \rightarrow \mathcal{M}_{m}(\mathbb{F})$ is a compound-commuting additive map if and only if either $\psi=0$, or $m=n$ and there exist a field homomorphism $\sigma: \mathbb{F} \rightarrow \mathbb{F}$, and invertible matrices $P, Q \in \mathcal{M}_{n}(\mathbb{F})$, with $C_{n-1}(P)=\mu P$ and $C_{n-1}(Q)=\mu^{-1} Q$ for some nonzero element $\mu \in \mathbb{F}$, such that

$$
\psi(A)=P A^{\sigma} Q \quad \text { for all } A \in \mathcal{M}_{n}(\mathbb{F}) \text {, or }
$$




$$
\psi(A)=P\left(A^{\sigma}\right)^{t} Q \quad \text { for all } A \in \mathcal{M}_{n}(\mathbb{F}) .
$$

Proof. The sufficiency is clear. We now consider the necessity. Suppose $\psi \neq 0$. It can be deduced from Lemma 2.5, applied on $\mathcal{T}_{n_{1}, \ldots, n_{k}}(\mathbb{F})=\mathcal{M}_{n}(\mathbb{F})$ (i.e., $k=1$ ), we get $m=n$ and $\psi$ is rank-one nonincreasing with $\psi\left(I_{n}\right)$ being of rank $n$. By Lemma 2.6, again applied on $\mathcal{T}_{n_{1}, \ldots, n_{k}}(\mathbb{F})=\mathcal{M}_{n}(\mathbb{F})$, there exist a scalar $\lambda \in\{0,1\}$, a nonzero field homomorphism $\sigma: \mathbb{F} \rightarrow \mathbb{F}$, and invertible matrices $P, Q \in \mathcal{M}_{n}(\mathbb{F})$ such that

$$
\psi(A)=P\left(\lambda A^{\sigma}+(1-\lambda)\left(A^{\sigma}\right)^{t}\right) Q \quad \text { for all } A \in \mathcal{M}_{n}(\mathbb{F}) .
$$

Since $\psi\left(C_{n-1}(A)\right)=C_{n-1}(\psi(A))$ for all $A \in \mathcal{M}_{n}(\mathbb{F})$, we have

$$
P^{-1} C_{n-1}(P) E_{i j}=E_{i j} Q C_{n-1}(Q)^{-1}
$$

for all $E_{i j} \in \mathcal{M}_{n}(\mathbb{F})$, and so, $P^{-1} C_{n-1}(P)=\mu I_{n}=Q C_{n-1}(Q)^{-1}$ for some nonzero element $\mu \in \mathbb{F}$. Hence, $C_{n-1}(P)=\mu P$ and $C_{n-1}(Q)=\mu^{-1} Q$. We are done.

By Lemma 2.5, Corollary 2.6 in [8] and Theorem 2.1 in [7], we have:

Theorem 2.13. Let $\mathbb{F}$ be a field, and let $m$ and $n$ be integers with $m, n \geqslant 3$. Then $\psi: \mathcal{S}_{n}(\mathbb{F}) \rightarrow \mathcal{S}_{m}(\mathbb{F})$ is a compound-commuting additive map if and only if $\psi=0$, or $m=n$ and there exist a nonzero field homomorphism $\sigma: \mathbb{F} \rightarrow \mathbb{F}$ and an invertible matrix $Q \in \mathcal{M}_{n}(\mathbb{F})$ with $C_{n-1}(Q)=\mu Q$ such that

$$
\psi(A)=\lambda Q A^{\sigma} Q^{t} \quad \text { for all } A \in \mathcal{S}_{n}(\mathbb{F}),
$$

where $\lambda, \mu \in \mathbb{F}$ are scalars such that $\lambda^{n-2} \mu^{2}=1$.

Proof. The sufficiency part can be easily checked. For the converse, we suppose $\psi \neq 0$. By Lemma 2.5, applied on $\mathcal{H}_{n}(\mathbb{F})=\mathcal{S}_{n}(\mathbb{F})$ (i.e., the field involution - on $\mathbb{F}$ is identity), we get $m=n$ and $\psi$ is a rank-one nonincreasing additive map with $\psi\left(I_{n}\right)$ being of rank $n$. By Corollary 2.6 in [8] and Theorem 2.1 in [7], we see that $\psi$ is of the following forms:

(a) $\psi(A)=\lambda Q A^{\sigma} Q^{t}$ for every $A \in \mathcal{S}_{n}(\mathbb{F})$, or

(b) $\psi(A)=P \zeta(A) P^{t}$ for every $A \in \mathcal{S}_{n}(\mathbb{F})$, only when $n=3$ and $\mathbb{F}=$ $\mathbb{Z}_{2}:=\{0,1\}$

Here, $Q \in \mathcal{M}_{n}(\mathbb{F})$ and $P \in \mathcal{M}_{3}\left(\mathbb{Z}_{2}\right)$ are invertible matrices, $\lambda \in \mathbb{F}$ is a nonzero scalar, $\sigma$ is a nonzero field homomorphism on $\mathbb{F}$, and $\zeta$ is a rank-one nonincreasing linear map on $\mathcal{S}_{3}\left(\mathbb{Z}_{2}\right)$ with $\zeta\left(I_{3}\right)$ being of rank 3 .

We first consider $\psi$ is of form (a). By the fact that $C_{n-1}(\psi(A))=\psi\left(C_{n-1}(A)\right)$ for $A \in \mathcal{S}_{n}(\mathbb{F})$, we have $(\lambda Q)^{-1} C_{n-1}(\lambda Q) D_{i j}=D_{i j} Q^{t} C_{n-1}\left(Q^{t}\right)^{-1}$ for all $1 \leqslant i, j \leqslant n$. Here, $D_{i i}=E_{i i}$, and $D_{i j}=-I_{n}+E_{i i}+E_{j j}+(-1)^{i+j+1}\left(E_{i j}+E_{j i}\right)$ when $i \neq j$. Thus, $(\lambda Q)^{-1} C_{n-1}(\lambda Q)=\mu^{-1} I_{n}=Q^{t} C_{n-1}\left(Q^{t}\right)^{-1}$ for some nonzero scalar $\mu \in \mathbb{F}$, and hence, $C_{n-1}(Q)=\mu Q$ and $\lambda^{n-2} \mu^{2}=1$.

Next, we suppose $\psi$ is of form (b). The compound-commuting of $\psi$ yields

$$
\zeta\left(C_{2}(A)\right)=D C_{2}(\zeta(A)) D^{t} \quad \text { for all } A \in \mathcal{S}_{3}\left(\mathbb{Z}_{2}\right),
$$


where $D=P^{-1} C_{2}(P) \in \mathcal{M}_{3}\left(\mathbb{Z}_{2}\right)$ is invertible. By (17), we see that $\zeta(A)$ is singular whenever $A \in \mathcal{S}_{3}\left(\mathbb{Z}_{2}\right)$ is singular. For, suppose $\operatorname{rank} \zeta\left(A_{0}\right)=3$ for some singular matrix $A_{0} \in \mathcal{S}_{3}\left(\mathbb{Z}_{2}\right)$. Then $\operatorname{rank} C_{2}\left(A_{0}\right) \leqslant 1$, but $\operatorname{rank} \zeta\left(C_{2}\left(A_{0}\right)\right)=$ $\operatorname{rank}\left(D C_{2}\left(\zeta\left(A_{0}\right)\right) D^{t}\right)=3$, which contradicts to the fact that $\psi$ is rank-one nonincreasing. Further, the linearity of $\zeta$ and $\operatorname{rank} \zeta\left(I_{3}\right)=3$ thus ascertain that $\operatorname{rank} \zeta\left(E_{i i}\right)=1$ for $i=1,2,3$, and $\operatorname{rank} \zeta\left(E_{i i}+E_{j j}\right)=2$ for all $1 \leqslant i \neq$ $j \leqslant 3$. Since $\zeta\left(E_{11}\right) \in \mathcal{S}_{3}\left(\mathbb{Z}_{2}\right)$ is of rank one, there exists an invertible matrix $U_{1} \in \mathcal{M}_{3}\left(\mathbb{Z}_{2}\right)$ such that $\zeta\left(E_{11}\right)=U_{1} E_{11} U_{1}^{t}$. Let

$$
\zeta\left(E_{22}\right)=U_{1}\left(\begin{array}{cc}
a_{1} & B_{1} \\
B_{1}^{t} & D_{1}
\end{array}\right) U_{1}^{t}
$$

with $a_{1} \in \mathbb{Z}_{2}, B_{1} \in \mathcal{M}_{1,2}\left(\mathbb{Z}_{2}\right)$ and $D_{1} \in \mathcal{S}_{2}\left(\mathbb{Z}_{2}\right)$. Clearly, $D_{1} \neq 0$. This is because if $D_{1}=0$, then, $\operatorname{since} \operatorname{rank} \zeta\left(E_{22}\right)=1$, we have $B_{1}=0$, and so, $\operatorname{rank} \zeta\left(E_{11}+E_{22}\right)<2$, a contradiction. Further, since $\operatorname{rank} \zeta\left(E_{22}\right)=1$, we conclude that $D_{1}$ is of rank one. Then there exists an invertible matrix $U_{2} \in \mathcal{M}_{2}\left(\mathbb{Z}_{2}\right)$ such that $D_{1}=U_{2} \operatorname{diag}(1,0) U_{2}^{t}$, and so,

$$
\begin{aligned}
\zeta\left(E_{22}\right) & =U_{1}\left(\begin{array}{cc}
a_{1} & B_{1} \\
B_{1}^{t} & U_{2}\left(\begin{array}{ll}
1 & 0 \\
0 & 0
\end{array}\right) U_{2}^{t}
\end{array}\right) U_{1}^{t} \\
& =U_{1}\left(\begin{array}{cc}
1 & 0 \\
0 & U_{2}
\end{array}\right)\left(\begin{array}{ccc}
a_{1} & b_{11} & b_{12} \\
b_{11} & 1 & 0 \\
b_{12} & 0 & 0
\end{array}\right)\left(\begin{array}{cc}
1 & 0 \\
0 & U_{2}^{t}
\end{array}\right) U_{1}^{t}
\end{aligned}
$$

with $b_{11}, b_{12} \in \mathbb{Z}_{2}$. Since $\operatorname{rank} \zeta\left(E_{22}\right)=1$, it follows that $b_{12}=0$ and $b_{11}^{2}=a_{1}$. Let

$$
U_{3}=U_{1}\left(\begin{array}{cc}
1 & 0 \\
0 & U_{2}
\end{array}\right)\left(\begin{array}{ccc}
1 & b_{11} & 0 \\
0 & 1 & 0 \\
0 & 0 & 1
\end{array}\right) \in \mathcal{M}_{3}\left(\mathbb{F}_{2}\right)
$$

Clearly, $U_{3}$ is an invertible matrix such that $\zeta\left(E_{i i}\right)=U_{3} E_{i i} U_{3}^{t}$ for $i=1,2$. Let

$$
\zeta\left(E_{33}\right)=U_{3}\left(\begin{array}{ll}
D_{2} & B_{2} \\
B_{2}^{t} & a_{2}
\end{array}\right) U_{3}^{t}
$$

with $a_{2} \in \mathbb{Z}_{2}, B_{2} \in \mathcal{M}_{2,1}\left(\mathbb{Z}_{2}\right)$ and $D_{2} \in \mathcal{S}_{2}\left(\mathbb{Z}_{2}\right)$. By the facts that $\operatorname{rank} \zeta\left(E_{33}\right)=$ 1 and $\operatorname{rank} \zeta\left(I_{3}\right)=3$, we conclude that $a_{2}=1$, and so, $B_{2} B_{2}^{t}=D_{2}$. Let

$$
U=U_{3}\left(\begin{array}{cc}
I_{2} & B_{2} \\
0 & 1
\end{array}\right) \in \mathcal{M}_{3}\left(\mathbb{F}_{2}\right) .
$$

It is clear that $U$ is invertible and $\zeta\left(E_{i i}\right)=U E_{i i} U^{t}$ for $i=1,2,3$.

Let $i, j, k$ be three distinct integers satisfying $1 \leqslant i, j, k \leqslant 3$. We denote $S_{i j}=$ $E_{i j}+E_{j i}$. Since $C_{2}\left(S_{i j}\right)=E_{4-k, 4-k}$, it follows from (17) that $C_{2}\left(\zeta\left(S_{i j}\right)\right)=$ $D \zeta\left(C_{2}\left(S_{i j}\right)\right) D^{t}=D \zeta\left(E_{4-k, 4-k}\right) D^{t}$ is of $\operatorname{rank}$ one, so $\operatorname{rank} \zeta\left(S_{i j}\right)=2$. Let $\zeta\left(S_{i j}\right)=U H_{i j} U^{t}$ with $H_{i j} \in \mathcal{S}_{3}\left(\mathbb{Z}_{2}\right)$ being of rank two. We want to claim that $H_{i j}=S_{i j}$. Since $S_{i j}+E_{i i}+E_{j j}$ is of rank one, we get $\operatorname{rank} \zeta\left(S_{i j}+E_{i i}+E_{j j}\right) \leqslant 1$. Suppose that $\zeta\left(S_{i j}+E_{i i}+E_{j j}\right)=0$. Then $\zeta\left(S_{i j}\right)=\zeta\left(E_{i i}\right)+\zeta\left(E_{j j}\right)=U\left(E_{i i}+\right.$ 
$\left.E_{j j}\right) U^{t}$. Thus, $\zeta\left(S_{i j}+E_{i i}+E_{k k}\right)=U\left(E_{j j}+E_{k k}\right) U^{t}$ is of rank two. Since $C_{2}\left(C_{2}\left(S_{i j}+E_{i i}+E_{k k}\right)\right)=S_{i j}+E_{i i}+E_{k k}$, it follows from (17) that $\zeta\left(S_{i j}+E_{i i}+\right.$ $\left.E_{k k}\right)=\zeta\left(C_{2}\left(C_{2}\left(S_{i j}+E_{i i}+E_{k k}\right)\right)\right)=0$. Hence, $\zeta\left(S_{i j}\right)=\zeta\left(E_{i i}\right)+\zeta\left(E_{k k}\right)$, and so, $\zeta\left(E_{k k}\right)=\zeta\left(E_{j j}\right)$, a contradiction. So, $\zeta\left(S_{i j}+E_{i i}+E_{j j}\right)=U\left(H_{i j}+E_{i i}+E_{j j}\right) U^{t}$ is of rank one. Let $H_{i j}=\left(h_{p q}\right) \in \mathcal{S}_{3}\left(\mathbb{Z}_{2}\right)$. Suppose $h_{k k} \neq 0$ (i.e., $h_{k k}=1$ ). Since $H_{i j}+E_{i i}+E_{j j}$ is of rank one, it follows that $h_{i i}+1=h_{i k}^{2}, h_{j j}+1=h_{j k}^{2}$ and $h_{i j}=h_{i k} h_{j k}$. Thus, we obtain $\operatorname{rank} H_{i j}=3$ for every $h_{i k}, h_{j k} \in \mathbb{Z}_{2}$, a contradiction. So, we conclude that $h_{k k}=0$. Further, since $H_{i j}+E_{i i}+E_{j j}$ is of rank one, it follows that $h_{i k}=h_{j k}=0$ and $\left(h_{i i}+1\right)\left(h_{j j}+1\right)=h_{i j}^{2}$. Suppose that $h_{i j}=0$. Since $\operatorname{rank} H_{i j}=2$, we conclude that $h_{i i}=1$ and $h_{j j}=1$, and so, $H_{i j}=E_{i i}+E_{j j}$. This leads to a contradiction because $\zeta\left(S_{i j}+E_{i i}+E_{j j}\right) \neq 0$. So, we conclude that $h_{i j}=1$, and so, $h_{i i}=h_{j j}=0$. Hence, $H_{i j}=S_{i j}$, as claimed. Consequently, we have

$$
\zeta(A)=U A U^{t} \quad \text { for all } A \in \mathcal{S}_{3}\left(\mathbb{Z}_{2}\right) .
$$

In view of $(\mathbf{b})$, we obtain $\psi(A)=Q A Q^{t}$ for all $A \in \mathcal{S}_{3}\left(\mathbb{Z}_{2}\right)$ where $Q=P U \in$ $\mathcal{S}_{3}\left(\mathbb{Z}_{2}\right)$ is an invertible matrix. We are done.

By Lemma 2.5, and the structural result of rank-one nonincreasing additive maps between Hermitian matrix spaces in [9, Theorem 2.7], [10, Main Theorem, p. 603] and [11, Theorem 2.1 and Remark 2.4], we have the following result.

Theorem 2.14. Let $\mathbb{F}$ be a field which possesses a proper involution ${ }^{-}$. Let $m$ and $n$ be integers with $m, n \geqslant 3$. Then $\psi: \mathcal{H}_{n}(\mathbb{F}) \rightarrow \mathcal{H}_{m}(\mathbb{F})$ is a compoundcommuting additive map if and only if $\psi=0$, or $m=n$ and there exist a nonzero field homomorphism $\sigma$ on $\mathbb{F}$ and an invertible matrix $P \in \mathcal{M}_{n}(\mathbb{F})$ with $C_{n-1}(P)=\mu P$ such that

$$
\psi(A)=\lambda P A^{\sigma} P^{*} \quad \text { for all } A \in \mathcal{H}_{n}(\mathbb{F}),
$$

where $\lambda, \mu \in \mathbb{F}$ are nonzero scalars such that $\bar{\lambda}=\lambda$ and $\lambda^{n-2} \mu \bar{\mu}=1$.

Acknowledgment. The author is grateful to Professor M. H. Lim for the valuable advice. The author would like to express his deep gratitude to the referees for their valuable suggestions.

\section{References}

[1] G. H. Chan and M. H. Lim, Linear preservers on powers of matrices, Directions in matrix theory (Auburn, AL, 1990). Linear Algebra Appl. 162/164 (1992), 615-626.

[2] G. H. Chan, M. H. Lim, and K. K. Tan, Linear preservers on matrices, Linear Algebra Appl. 93 (1987), 67-80.

[3] W. L. Chooi, Rank-one nonincreasing additive maps between block triangular matrix algebras, Linear and Multilinear Algebra 58 (2010), no. 6, 715-740.

[4] W. L. Chooi and M. H. Lim, Coherence invariant mappings on block triangular matrix spaces, Linear Algebra Appl. 346 (2002), 199-238.

[5] A. Fošner and P. Šemrl, Additive maps on matrix algebras preserving invertibility or singularity, Acta Math. Sin. (Engl. Ser.) 21 (2005), no. 4, 681-684. 
[6] G. Frobenius, Über die Darstellung der endlichen Gruppen durch Lineare Substitutionen, Stizungsber. Deustch. Akad. Wiss. Berlin (1897), 994-1015.

[7] B. Kuzma and M. Orel, Additive mappings on symmetric matrices, Linear Algebra Appl. 418 (2006), no. 1, 277-291.

[8] M. H. Lim, Rank-one nonincreasing additive mappings on second symmetric product spaces, Linear Algebra Appl. 402 (2005), 263-271.

[9] _ Additive mappings between Hermitian matrix spaces preserving rank not exceeding one, Linear Algebra Appl. 408 (2005), 259-267.

[10] M. Orel and B. Kuzma, Additive maps on Hermitian matrices, Linear Multilinear Algebra 55 (2007), no. 6, 599-617.

[11] _ Additive rank-one nonincreasing maps on Hermitian matrices over the field GF $\left(2^{2}\right)$, Electron. J. Linear Algebra 18 (2009), 482-499.

[12] S. Pierce et al., A survey of linear preserver problems, Linear and Multilinear Algebra 33 (1992), no. 1-2. Gordon and Breach Science Publishers, Yverdon, 1992. pp. 1-129.

[13] R. Sinkhorn, Linear adjugate preservers on the complex matrices, Linear and Multilinear Algebra 12 (1982/83), no. 3, 215-222.

[14] X. M. Tang, Additive rank-1 preservers between Hermitian matrix spaces and applications, Linear Algebra Appl. 395 (2005), 333-342.

[15] X. M. Tang and X. Zhang, Additive adjoint preservers between matrix spaces, Linear Multilinear Algebra 54 (2006), no. 4, 285-300.

[16] Z. X. Wan, Geometry of Matrices, World Scientific, Singapore, 1996.

[17] X. Zhang, X. M. Tang, and C. G. Cao, Preserver Problems on Spaces of Matrices, Science Press, Beijing, 2007.

Institute of Mathematical Sciences

UNIVERSITY OF MALAYA

50603 Kuala Lumpur, Malaysia

E-mail address: wlchooi@um.edu.my 